San Jose State University

SJSU ScholarWorks

Master's Theses

Master's Theses and Graduate Research

Fall 2016

\title{
Elucidating the Role of Mastermind-like 1 in the Degradation of Myocyte Enhancer Factor 2C
}

Azia S. Evans

San Jose State University

Follow this and additional works at: https://scholarworks.sjsu.edu/etd_theses

\section{Recommended Citation}

Evans, Azia S., "Elucidating the Role of Mastermind-like 1 in the Degradation of Myocyte Enhancer Factor 2C" (2016). Master's Theses. 4751.

DOI: https://doi.org/10.31979/etd.e48y-u8fx

https://scholarworks.sjsu.edu/etd_theses/4751

This Thesis is brought to you for free and open access by the Master's Theses and Graduate Research at SJSU ScholarWorks. It has been accepted for inclusion in Master's Theses by an authorized administrator of SJSU ScholarWorks. For more information, please contact scholarworks@sjsu.edu. 


\title{
ELUCIDATING THE ROLE OF MASTERMIND-LIKE 1 IN THE DEGRADATION
} OF MYOCYTE ENHANCER FACTOR 2C

\author{
A Thesis \\ Presented to \\ The Faculty of the Department of Biological Sciences \\ San José State University \\ In Partial Fulfillment \\ of the Requirements for the Degree \\ Master of Science
}

by

Azia S. Evans

December 2016 
(C) 2016

Azia S. Evans

ALL RIGHTS RESERVED 
The Designated Thesis Committee Approves the Thesis Titled

ELUCIDATING THE ROLE OF MASTERMIND-LIKE 1 IN THE DEGRADATION OF MYOCYTE ENHANCER FACTOR 2C

by

Azia S. Evans

APPROVED FOR THE DEPARTMENT OF BIOLOGICAL SCIENCES

SAN JOSÉ STATE UNIVERSITY

DECEMBER 2016

$\begin{array}{ll}\text { Dr. J. Brandon White } & \text { Department of Biological Sciences } \\ \text { Dr. Cleber Ouverney } & \text { Department of Biological Sciences } \\ \text { Dr. Alberto Rascón } & \text { Department of Chemistry }\end{array}$




\begin{abstract}
Mastermind-like 1 (MAML1) is well characterized for its involvement in the Notch signaling pathway where it functions as a transcriptional co-activator and facilitates the degradation of the Notch intracellular domain (NICD). MAML1 has recently been implicated as a multifunctional protein that interacts with a variety of other signaling pathways, including Wnt- $\beta$-catenin, NF- $\kappa \mathrm{B}$, and MEF2C (myocyte enhancer factor $2 \mathrm{C}$ ). $\mathrm{MEF} 2 \mathrm{C}$ is a member of the family of transcription factors involved in muscle cell differentiation and proliferation. MAML1 has previously been shown to activate MEF2C transcription. Given that MAML1 is responsible for degradation of the NICD, we wanted to know if MAML1 could also induce degradation of MEF2C. Here we show that full length MAML1 induces degradation of MEF2C. MEF2C is not degraded when co-expressed with MAML1 deletion constructs, MAML1 1-301 and MAML1 $\triangle 75-300$, suggesting the entire c-terminus is needed for degradation. In addition, treatment with the proteasome inhibitor MG132 stabilized MEF2C expression both in the absence and presence of MAML1 when compared to dimethyl sulfoxide (DMSO) controls. Immunopreciptations of MEF2C in the presence of MAML1 with MG132 treatment showed an increase in the amount of ubiquitin detected. Serine 98 and 110 in MEF2C appear to be essential for ubiquitination. In order to determine if S98 and S110 are required for MAML1 degradation of MEF2C, we cloned the double serine mutant MEF2C S98A S110A. However, co-expression of the mutant with MAML1 still resulted in decreased protein levels. Our results showed MAML1-induced degradation of MEF2C occurs through the proteasomal pathway and that S98 and S110 are not required for degradation.
\end{abstract}




\section{TABLE OF CONTENTS}

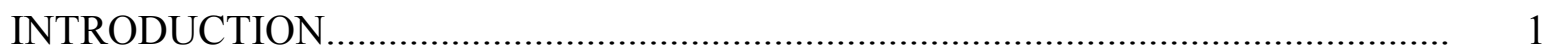

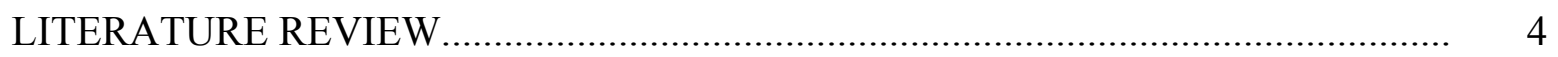

Mastermind Family of Transcription Co-activators..................................... 4

Mastermind-like 1's Role in the Notch Signaling Pathway............................. 5

MAML1 Functions Across Multiple Signaling Pathways.................................. 6

MEF2C Signaling.............................................................................. 8

Ubiquitin- Proteasome Dependent Protein Degradation................................. 11

EXPERIMENTAL PROCEDURES................................................................ 12

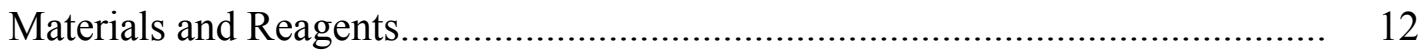

Generation of MEF2C Mutant plasmids................................................... 13

Maxi Prep Plasmid Purification............................................................. 15

Transfection of Mammalian Cells........................................................... 16

Degradation Assays.......................................................................... 17

MEF2C Immunoprecipitations (IP) .................................................. 17

SDS-PAGE Preparation and Western Blotting ........................................ 18

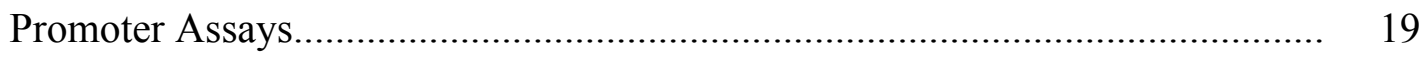

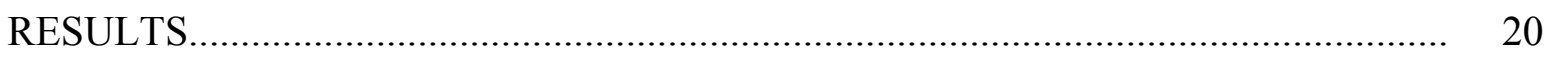

Co-expression of MEF2C and Mastermind-like 1 Leads to Reduced MEF2C Protein Levels.................................................................................... 20

Both TADs of Mastermind-like 1 are Required for Degradation to Occur......... 21 
Mastermind-like 2 and Mastermind-like 3 Elicit Varying Effects on MEF2C

Protein Levels

MG132 Treatment Recovers MEF2C Protein Levels......................................... 24

Detection of Ubiquitin on MEF2C................................................................. 26

Mastermind Does Not Induce Degradation of MEF2C in Colorectal Cancer

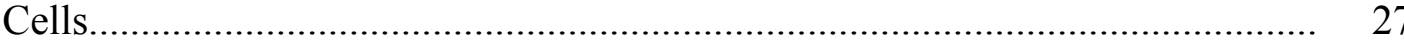

MEF2C Mutants Do Not Prevent MAML1 Induced Degradation........................ 31

Effects on MEF2C Target Promoter Activity...................................................... 31

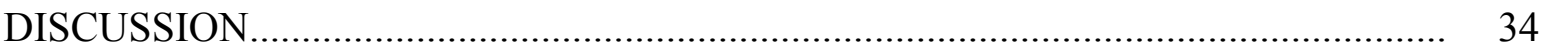

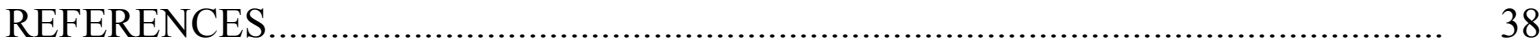




\section{LIST OF TABLES}

Table 1: $\quad$ Site-directed mutagenesis primer sequences.......................... 13

Table 2: $\quad$ Lipofectamine 2000 transfection cell density...................... 16 


\section{LIST OF FIGURES}

Figure 1. Full length MAML1 reduces the half-life of MEF2C and results in decreased protein levels.................................... 3

Figure 2. Characteristic domains of mastermind-like co-activators............ 4

Figure 3. MAML1 induced degradation of MEF2C.................... 20

Figure 4. MAML1 deletion construct deficient in TAD1 or TAD2............ 21

Figure 5. Co-expression of MEF2C with MAML1 deletion constructs......... 22

Figure 6. MAML2 and MAML3 do not induce degradation of MEF2C........ 23

Figure 7. MG132 treatment recovers MEF2C protein levels................ 25

Figure 8. Detection of ubiquitin on MEF2C treated with MG132........... 26

Figure 9. MAML1 does not induce degradation of MEF2C in HCT116 cells.. 28

Figure 10. MAML1 deletion constructs are unable to induce degradation of native MEF2C..........................................

Figure 11. MAML2 and MAML3 are unable to induce degradation of MEF2C in $\mathrm{HCT} 116$ cells........................................... 30

Figure 12. MAML1 can induce degradation of MEF2C S98A S110A.......... 31

Figure 13. Effects on MEF2 target promoter activity..................... 33 


\section{ABBREVIATIONS}

APC/C: Anaphase promoting complex/ cyclosome

CBF-1: Centromere binding factor 1

CBP: CREB binding protein

CDC20: Cell division cycle 20 protein

CDK8: Cyclin-dependent kinase 8

CSL: CBF-1, suppressor of hairless, and Lag-1

DMEM: Dulbecco’s Modified Eagles Medium

DMSO: Dimethyl sulfoxide

GAPDH: Glyceraldehyde 3-phosphate dehydrogenase

GSK3 $\beta$ : Glycogen synthase kinase-3 beta

Hes1: Hairy and enhancer of split-1

Hey1: Hes-related family BHLH transcription factor with YRPW motif 1

IP: Immunoprecipitation

MADS: MCM1, agamous, deficiens, and serum-response factor

MAML: Mastermind-like protein

MAML1: Mastermind-like protein 1

MAML2: Mastermind-like protein 2

MAML3: Mastermind-like protein 3

MEF2: Myocyte enhancer factor 2

MEF2C: Myocyte enhancer factor 2C

MRF: Myogenic regulatory factor 
Mrf4: Myogenic regulatory factor 4

Myf5: Myogenic factor 5

NICD: Notch intracellular domain

NF-кB: Nuclear factor kappa B

RelA: V-Rel avian reticuloendotheliosis viral oncogene homolog A

SDS-PAGE: Sodium dodecyl sulfate- polyacrylamide gel electrophoresis

TAD: Transcription activating domain

TCF: T cell factor

Wnt: Wingless-type MMTV integration site family member 


\section{INTRODUCTION}

Myocyte enhancer factor 2C (MEF2C) is a human transcription factor first identified for its importance in muscle cell differentiation $(1,2)$. Recent studies have demonstrated MEF2C is an essential regulatory transcription factor associated with multiple developmental pathways and disease abnormalities (3-7). MEF2C belongs to the MADS box family of transcription factors, which includes MEF2A-D. These transcription factors are non-myogenic alone but, when expressed with other myogenic regulatory factors (MRFs), they elicit myogenic functions to allow the differentiation of mesenchymal stem cells to myofibers $(1,8)$.

Previous studies have implicated Mastermind-like 1 (MAML1) as an important co-activator for MEF2C-mediated transcription (8). Unlike MEF2C, MAML1 does not interact with DNA directly but associates with other essential DNA-binding transcription factors, allowing efficient transcription initiation. MAML1 was originally identified as an essential co-activator for the Notch signaling pathway, where it associates with the Notch intracellular domain (NICD) and centromere-binding factor 1 (CBF-1) to allow initiation of Notch target genes such as Hes1, Hes5, and Hey1 (9). MAML1 is also required to facilitate the turnover of NICD in the nucleus. MAML1 has been shown to interact with CDK8 and CBF-1 to facilitate the phosphorylation-dependent ubiquitination of NICD (10). Notch target genes are generally implicated for their importance in growth and differentiation. Therefore, this additional regulatory step is crucial to keep expression under control. Abnormalities in these signaling pathways have been shown to lead to various cancers $(11,12)$. 
Based on MAML1's known function in the Notch signaling pathway and the discovery of MAML1 as a co-activator for MEF2C, we wanted to know if MAML1 was capable of inducing degradation of MEF2C. Preliminary results generated by our lab show MAML1 does induce MEF2C degradation and we sought to determine whether degradation was occurring through the ubiquitin-proteasome pathway or through lysosomal degradation. Our lab demonstrated that MAML1 decreased the half-life of MEF2C by 80 minutes determined by cyclohexamide pulse chase (Figure $1 \mathrm{~A}, \mathrm{~B}$ ) (13). Additionally, MEF2C protein levels were lower in the presence of MAML1 at time point zero of cylohexamide treatment, indicating that MAML1 has the ability to maintain reduced levels of MEF2C. Our lab also previously found both transcriptional activation domains (TAD) of MAML1 are required for degradation to occur (Figure 1 C, D) (13).

We hypothesized that MAML1 would facilitate the turnover of MEF2C through phosphorylation-dependent ubiquitination and target MEF2C to be degraded by the $26 \mathrm{~S}$ proteasome. In addition to identifying MEF2C's degradation pathway, we hoped to determine the effects of MAML1-induced MEF2C degradation on MEF2 promoter activity. 
$\mathbf{A}$
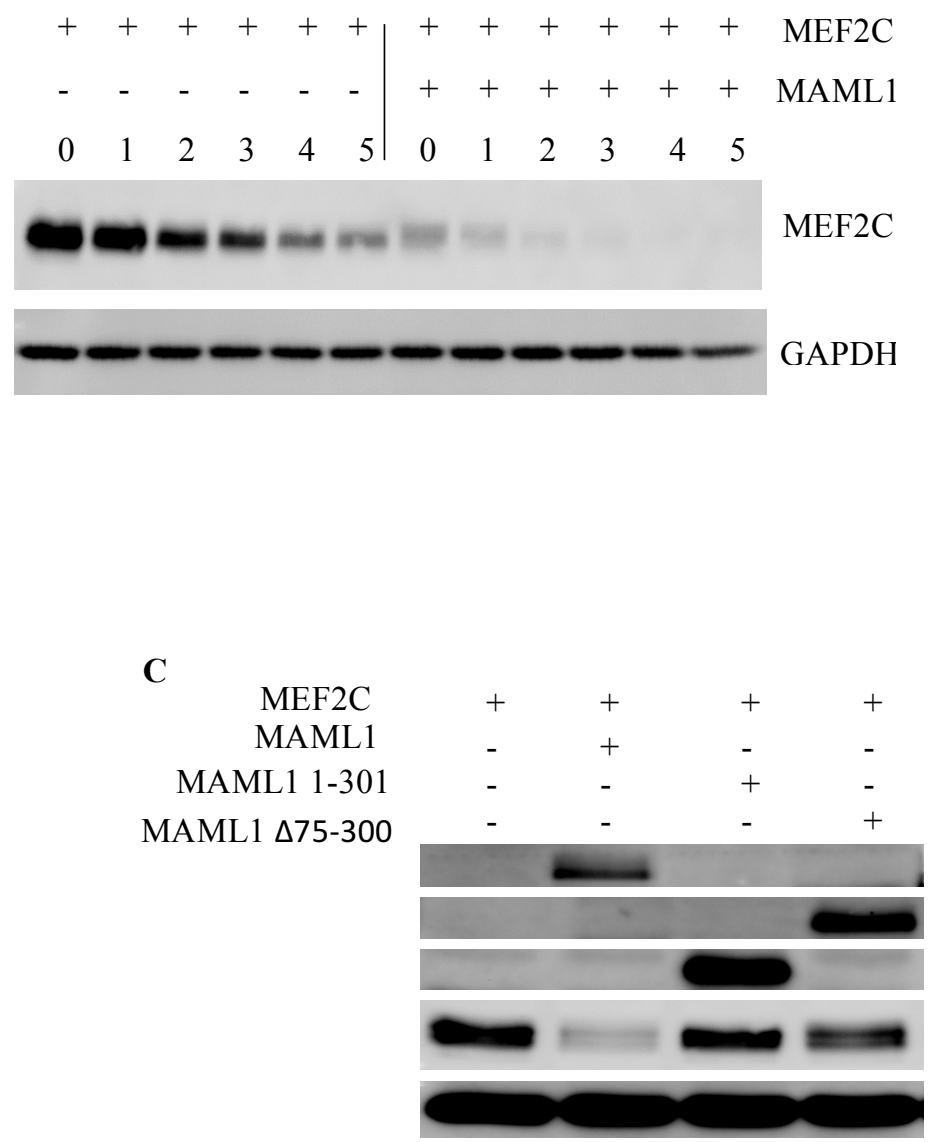

D

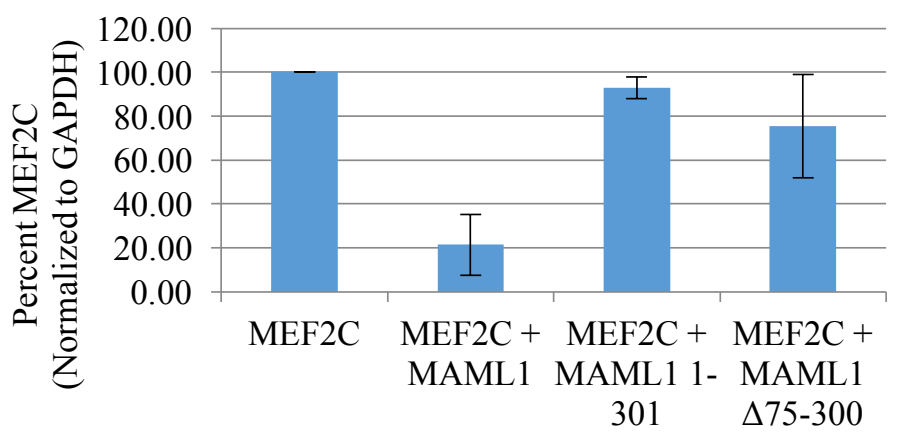

Figure 1. Full length MAML1 reduces the half-life of MEF2C and results in decreased protein levels. (A) Cyclohexamide pulse chase of HeLa cells expressing MEF2C in the absence and presence of MAML1. (B) The half-life of MEF2C with and without MAML1, determined from cyclohexamide pulse chase data (A) and shown in minutes. (C) MEF2C expressed alone or with MAML1 or MAML1 deletion constructs. (D) Quantification of MEF2C protein levels shown in C and normalized to GAPDH. 


\section{LITERATURE REVIEW}

\section{Mastermind Family of Transcription Co-activators}

Mastermind was first identified as a member of the Notch signaling pathway in Drosophila melanogaster. Like many developmental genes, this pathway has remained conserved across many species (14). The mammalian homolog to Drosophila mastermind is the mastermind-like (MAML) family, which is composed of three members, MAML1, MAML2, and MAML3 (Figure 2) $(9,15)$. All members of the MAML family are characterized by the presence of one basic and two acidic amino acid domains. The N-terminal basic domain, located within the first 14-78 amino acids in MAML1, is required for binding NICD and $\operatorname{MEF} 2 \mathrm{C}(8,15)$. TAD1 begins directly after the basic domain at amino acid 79 to 300 , while a region within TAD1 is required for binding of p300 and CREB-binding protein $(\mathrm{CBP})(8,15,16)$. The C-terminal TAD2 is required for promoter activation, though it is uncertain what co-factors interact with this region and are responsible for transcriptional activation (17).

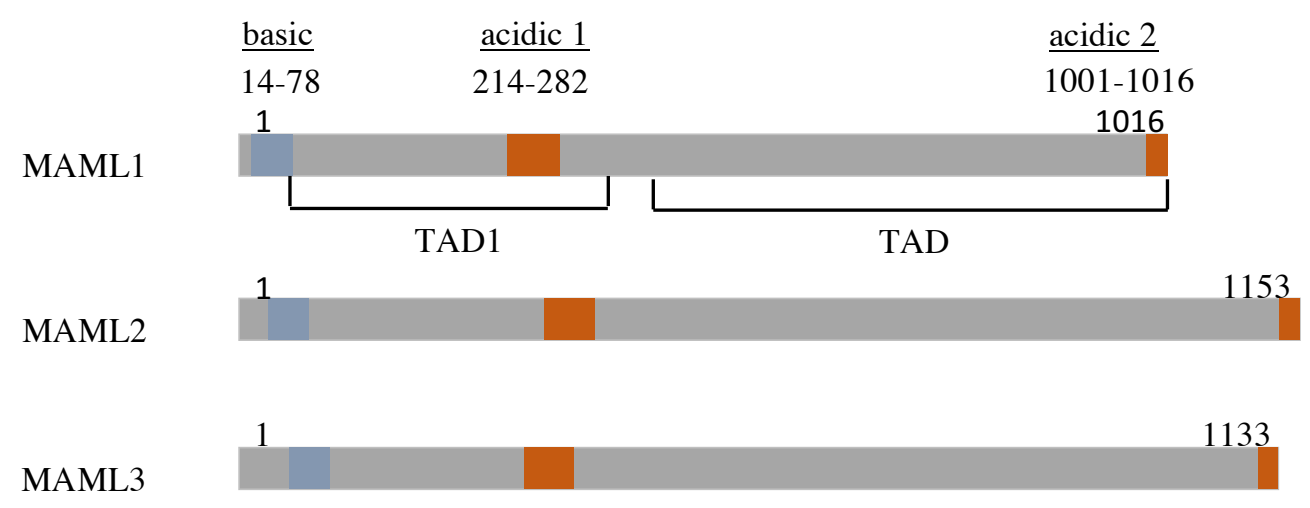

Figure 2. Characteristic domains of mastermind-like co-activators. The MAML family of transcriptional co-activators have two transcriptional activation domains TAD1 and TAD2. MAML1, MAML2, and MAML3 all contain one basic and two acidic amino acid domains. 


\section{Mastermind-like 1's Role in the Notch Signaling Pathway}

Notch signaling is an evolutionarily conserved pathway essential for development and proliferation (18). Abnormalities in this signaling pathway lead to disease progression, often resulting in the development of cancers (12). Notch signaling is initiated when a Notch receptor on the surface of the receiving cell binds a Delta/Jagged ligand on the inducing cell. This interaction between receptor and ligand initiates cleavage of the Notch intracellular domain (NICD) by $\gamma$-secretase. This cleavage event releases NICD from the extracellular portion of the receptor, allowing it to translocate to the nuclease where it interacts with a CSL (CBF-1, Suppressor of hairless, and Lag-1) family member of transcription factors and MAML1 $(10,19,20)$. Assembly of the NICD transcriptional complex results in transcription of Notch target genes such as Hes 1, Heyl (Hairy/enhancer of split), $c-M y c$, and $p 21(10,21-23)$. Hes 1 and Hey1 are primarily known to be transcriptional repressors important in neural development, while p21 is an important factor involved in cell cycle arrest (21-23). Many of the known Notch target genes have been implicated in pathways involved in development and cell proliferation; therefore, it is essential that they be tightly regulated. Uncontrolled Notch activity has been shown to lead to a variety of cancers $(10,20)$.

MAML1 has been shown to be a co-activator for NICD and the corresponding CSL family of transcription factors by aiding in the recruitment of p300 (10). In the absence of MAML1, transcriptional activity of NICD is drastically reduced. However, reconstituted MAML1 significantly up regulates the transcription of the reporter gene $(10,19,21)$. Association of MAML1 with NICD and CBF-1 (a CSL transcription factor) 
is responsible for activating Notch target transcription. Specifically, the N-terminus of MAML1 is required for the interaction with the NICD, while the C-terminus is essential for the recruitment of remaining cofactors and activation of the transcriptional complex $(16,21)$.

While MAML1 has been shown to significantly up regulate the activity of the Notch transcriptional complex, it has also been shown to be essential in regulating the turnover of NICD through a phosphorylation-dependent mechanism (10). MAML1 and Cyclin-dependent kinase 8 (CDK8) work jointly to facilitate phosphorylation of NICD as

a method to ensure tight regulation of Notch target genes (10). Phosphorylation of NICD signals it for ubiquination by the ubiquitin ligase Fbw7 and proteasomal degradation (10, 24). Whether MAML1 activates the transcriptional complex or inhibits it through inducing NICD turnover appears to be dependent on the time elapsed following the initial transcription activation event $(10,24)$. While this mechanism is not fully understood, research suggests MAML1 functions as an activator initially but later recruits CDK8 and facilitates turnover of the complex. MAML1 regulation of NICD turnover is essential for maintenance of the signaling pathway. This ensures that Notch target genes, known to play an important role in development and tissue differentiation, are tightly regulated; preventing consequences often associated with uncontrolled cell growth and proliferation.

\section{MAML1 Functions Across Multiple Signaling Pathways}

MAML1 has been implicated as an important co-factor in multiple signaling

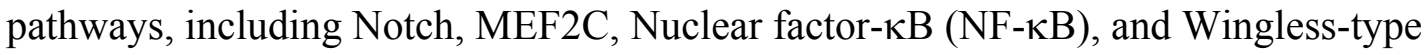
MMTV integration site family member (Wnt)- $\beta$-catenin. It has been suggested that 
MAML1 may play an important role in maintaining communication and balance across these signaling pathways during developmental processes $(8,17,25-28)$.

MAML1 regulates the NF- $\kappa B$ signaling pathway through co-activation of RelA and induced degradation of the inhibitor I $\mathrm{I} \mathrm{B} \alpha$. NF- $\mathrm{NB}$ signaling is associated with many diverse functions including cell proliferation, inflammation, and innate and adaptive immune responses (27). Regulation of this pathway is highly important for the prevention of many diseases such as arthritis, diabetes, cancer, and various viral

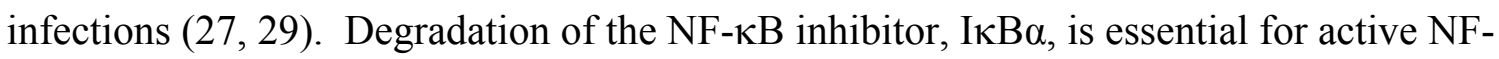
$\kappa \mathrm{B}$ signaling events. MAML1 enhances NF- $\mathrm{KB}$ signaling by facilitating modifications in

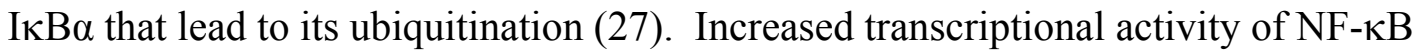
target genes has not only been attributed to the degradation of $\mathrm{I} \mathrm{B} \alpha \boldsymbol{\alpha}$ but also to the interaction between MAML1 and the RelA subunit of NF-kB (27). Evidence indicates MAML1 interacts with RelA, resulting in co-localization of MAML1 and NF-kB in the nucleus. MAML1 causes the same pattern formation in the nucleus during Notch signaling and MEF2C activation, suggesting the formation of a transcriptional complex $(8,10,27)$.

MAML1 plays an important role in the Wnt- $\beta$-catenin signaling pathway as a coactivator for $\beta$-catenin and the associated T-cell factor (TCF) transcription factor (30). Additionally, the Wnt signaling pathway up regulates the expression of MAML1 through the down regulation of GSK3 $\beta$. GSK3 $\beta$ is associated with inhibition of MAML1 transcriptional activity through direct interaction. Wnt signaling inhibits expression of GSK3 $\beta$, resulting in the up regulation of MAML1 and increased transcriptional activity 
of MAML1 target pathway (25). The Wnt pathway has been implicated as a potential regulator of the crosstalk between Notch and various other signaling pathways through MAML1 regulation $(25,31)$. In contrast, smaller niche environments, such as stem cells, show evidence that Wnt and Notch signaling pathways oppose each other (31). This relationship has not been fully elucidated; however, the distribution of MAML1 may play an important role in the regulation of these pathways and their effects on cell proliferation.

\section{MEF2C Signaling}

MEF2C belongs to the Myocyte Enhancer Factor 2 (MEF2) family of transcription factors originally identified as muscle-specific genes due their up regulation during myoblast differentiation $(1,32)$. MEF2 family contains four members, MEF2AD, which all contain two conserved domains, the MADS (MCM1, Agamous, Deficiens, and serum-response factor) box domain and MEF2 domain. The MADS domain is located within the first 55 amino acids, while the MEF2 domain is located within the 27 amino acids downstream of the MADS box (32). The MEF2 protein domain is responsible for recognizing the AT rich sequence $\left(\mathrm{CTA}(\mathrm{A} / \mathrm{T})_{4} \mathrm{TAG}\right)$ known as the MEF2 element within the promoter E-box of target genes such as Myogenin, MyoD, and Desmin $(1,32,33)$. The MADS and MEF domains are responsible for allowing protein dimerization and DNA binding (8). MEF2 proteins have been shown to bind their target promoter element as either homo or heterodimers dependent on the specific cell type.

This variation in binding activity may influence the transcriptional effects on target gene expression $(32,34)$. Other members of the MEF2 family are expressed ubiquitously, 
while MEF2C is believed to be only highly expressed in skeletal muscle and the cerebral cortex $(1,2,34)$.

Skeletal muscle formation originates when undifferentiated myoblasts exit the cell cycle and fuse to form multinucleated myotubes. This differentiation process is associated with the activation of muscle-specific genes, such as myogenic regulatory factors (MRFs), SRF, and MEF2. Additionally, myogenesis relies on the down regulation of genes associated with growth and division $(8,33)$. MRFs, including MyoD, myogenin, Myf5, and MRF4 form heterodimers with E proteins and interact with the Ebox region of a target gene. Unlike MEF2 proteins, all MRFs have independent myogenic activity (8).

MAML1 has been shown to be an essential co-activator for MEF2C both in overexpressed cell culture systems and during the myogenic differentiation profile. Research has proven that $M A M L 1$ knock out mice have drastically reduced survival rates and exhibit muscular dystrophy like symptoms. The hallmarks of this knock out show significant decrease in size and weight, as well as severe alterations to skeletal muscle when compared to wild-type mice (8). Mouse embryonic fibroblasts (MEFs) can be transduced with MyoD to differentiate into distinct myotubes. However in MAML1 knock out MEFs, this differentiation is unable to occur (8). Shen et al. found a distinct correlation between the amount of MAML1 transduced in $\mathrm{C} 2 \mathrm{C} 12$ cells and the reporter activation of an artificial MEF2 promoter. Indicating MAML1 and MEF2C act cooperatively to activate gene expression. Additionally MAML1 and MEF2C have been shown to co-localize in the nuclear foci formation that is indicative of NICD and 
MAML1 in the nucleus $(8,10)$. Similar to MAML1's role in Notch signaling, both TAD1 and TAD2 are required for full activation of the MEF2C promoter. Importantly when MAML1 and MEF2C are co-expressed there is a molecular weight shift in the western blot, indicating MAML1 may be inducing a post-translational modification on MEF2C (8).

When Notch signaling is activated concurrent to MEF2C transcription, it results in a decrease of transcriptional activity to basal levels suggesting Notch has the power to sequester MAML1 for it's own needs (8). Interestingly, Notch signaling has been shown to both activate and inhibit myogenesis when activated at specific time points during differentiation $(8,35,36)$. Considering MAML1 regulates the turnover of NICD in the nucleus, we must examine the possibility of a similar function with MEF2C. Minimal research has been done on this topic, however it has been shown that MEF2C transcriptional activity is tightly regulated but the involvement of MAML1 has yet to be discovered $(37,38)$.

Recent research suggests MEF2C plays an important role in cell cycle regulation $(37,38)$. MEF2C target genes are often involved in preventing cell proliferation due to their role in myogenesis and exit from the cell cycle. Therefore, MEF2C must be degraded in order for cell proliferation to occur. One study suggests Anaphase promoting complex/ Cyclosome $(\mathrm{APC} / \mathrm{C})$ ubiquitin ligase is responsible for regulating $\mathrm{MEF} 2 \mathrm{C}$ levels allowing for cell cycle progression (37). Badodi et al. shows a phosphorylation dependent mechanism allowing for MEF2C ubiquitiniation by $\mathrm{APC} / \mathrm{C}$ relies on the 
phosphorylation of serines 98 and 110 through a Cell division cycle 20 (CDC20) mechanism.

\section{Ubiquitin- Proteasome Dependent Protein Degradation}

The Ubiquitin-Proteasome pathway is closely associated with regulation of transcription factors in the nucleus. Ubiquitin ligases have been shown to interact with a number of co-factors to facilitate the turn-over of transcriptional complexes, keeping gene expression under tight regulation (39). One common mechanism of targeted ubiquitination is through modification of the substrate, usually in the form of phosphorylation (40). Once the desired substrate has been identified by either phosphorylation or recognition of the degron, ubiquitin is covalently linked through a series of enzymatic reactions. First the ubiquitin-activating enzyme (E1) transfers ubiquitin to the ubiquitin-conjugating enzyme (E2). E2 then associates with the ubiquitin-ligase (E3) specific to the substrate and ubiquitin is covalently attached (41). In mammalian cells, there are multiple variations of E2 and E3 enzymes, allowing for greater specificity during the protein degradation process. Once the target protein has been ubiquitinated, it is then directed to the $26 \mathrm{~S}$ proteasome for degradation. This process is extremely important for regulating transcription, allowing for increased sensitivity to cellular signals while preventing the overexpression of genes needed for growth and proliferation $(41,42)$. 


\section{EXPERIMENTAL PROCEDURES}

\section{Materials and Reagents}

HeLa cells and HEK-293 cells were purchased from American Type Culture Collection (ATCC). HCT116 cells were obtained from the UCB Cell Culture Facility, supported by the University of California Berkeley. All cell lines were maintained in Dulbecco's Modified Eagles Medium (DMEM) containing 10\% fetal bovine serum and $1 \%$ antibiotic-antimycotic at $37^{\circ} \mathrm{C}$ with $5 \% \mathrm{CO}_{2}$ injection. DMEM and $100 \mathrm{X}$ antibioticantimycotic were purchased from Mediatech (Manassas, VA).

Lipofectamine 2000, Opti-MEM, Protein A/G Magnetic Beads, LR Clonase, and 100X Halt Protease Cocktail were purchased from Thermo Fisher Scientific (Waltham, MA). Quickchange II XL Site-Directed Mutagenesis kit and XL10-Gold ultracompetent cells purchased from Agilent Technologies (Santa Clara, CA). GenCatch Maxi Prep columns were purchased from Epoch Life Sciences (Missouri City, TX). P1, P2, P3, QBT, and QF maxi prep buffers purchased from Qiagen (Hilden, Germany). The Dual Luciferase Reporter Assay System and PureYield Plasmid Miniprep kit were purchased from Promega (Madison, WI).

Sodium dodecyl sulfate-polyacrylamide gel electrophoresis (SDS-PAGE) buffers were prepared as follows: $4 \mathrm{x}$ lower buffer $(375 \mathrm{~mL}$ of $2 \mathrm{M}$ Tris at $\mathrm{pH} 8.8,10 \mathrm{ml}$ of $10 \%$ SDS, $115 \mathrm{ml}$ MilliQ H2O), 4x upper buffer (125 ml of 2M Tris at $\mathrm{pH} 6.8,10 \mathrm{ml}$ of 10\% SDS, $365 \mathrm{ml}$ of MilliQ H2O).

Original MAM1 plasmids and mutant MAM1 constructs were prepared by Dr. J. Brandon White using Invitrogen's Gateway technology. The mastermind plasmids 
(MAML1, MAML2, MAML3, MAML1 1-301, and MAML1 475-300) were all cloned into the pCS2 6XMyc by Dr. J. Brandon White using Gateway technology. HA-MEF2C was purchased from addgene as pcDNA3.1-MEF2C-HA. Myc-MEF2C was cloned into the pCS2 6XMyc vector using Gateway Cloning Technology with LR Clonase.

Imaging of all western blots was performed on the ImageQuant LAS4000, from GE Healthcare (Little Chalfont, UK). Promoter assay readings were obtained using a Veritas Microplate Luminometer from Promega (Madison, WI). All data analysis was done using Microsoft Excel and ImageQuant TL software.

\section{Generation of MEF2C Mutant plasmids}

MEF2C mutant constructs (S98A, S110A, and S98A S110A) were made in both the pcDNA3.1 and pCS2 backbone plasmids using the QuickChange II XL Site-Directed Mutagenesis kit. Forward and reverse primers were designed to mutate both serine 98 and serine 110 independently to alanine (Table 1). Single mutant constructs were generated first and subsequently used as the DNA template for making the double mutant construct.

Table 1: Site-directed mutagenesis primer sequences

\begin{tabular}{|c|l|}
\hline \multicolumn{2}{|l|}{ MEF2C Mutant Primer sequences } \\
\hline $\begin{array}{c}\text { MEF2C } \\
\text { Mutant }\end{array}$ & Forward primer \\
\hline S98A & GGGCCTCAATGGCTGTGACGCCCCAGATCCCGATGCACAGACGATTC \\
\hline S110A & GCAGACGATTCAGTAGGTCACGCCCCTGAGTCTGAGGACAAGTACAGG \\
\hline
\end{tabular}

Following completion of the mutagenesis PCR, all samples were incubated with 1 $\mu \mathrm{L}$ Dpn1 restriction enzyme at $37^{\circ} \mathrm{C}$ for 1.5 hours to digest methylated template DNA. 
Samples were subjected to heat inactivation of the enzyme at $95^{\circ} \mathrm{C}$ for 5 minutes prior to transformation into XL10-Gold ultracompetent cells. Two microliters of $\beta$ mercaptoethanol ( $\beta$-ME) was added to $40 \mu \mathrm{L}$ of competent cells and incubated on ice for 10 min. Next, $5 \mu \mathrm{L}$ of the PCR reaction was added to the $\beta$-ME/ competent cell mixture and incubated on ice for $30 \mathrm{~min}$. The transformation mixture was heat shocked at $42^{\circ} \mathrm{C}$ for 30 seconds and left on ice for $2 \mathrm{~min}$. Following transformation, the competent cells were recovered in NZY broth shaking at $225 \mathrm{rpm}$ and $37^{\circ} \mathrm{C}$ for $1.5 \mathrm{~h}$. The transformation was then spun down and plated on LB-ampicillin plates and incubated at $37^{\circ} \mathrm{C}$ overnight. Following incubation of the site-directed mutagenesis transformation, colonies were picked and grown in $5 \mathrm{~mL}$ of LB-ampicillin broth overnight. Cultures were then mini-prepped using the Promega PreYield Plasmid miniprep system. Five hundred microliters of each culture was saved as a bacterial stock to maxi prep at a later time if mutagenesis was correct. Five milliliter cultures were centrifuged at $3000 \mathrm{rpm}$ for 5 min. Bacterial pellets were resuspended in $600 \mu \mathrm{L}$ of Tris-EDTA (TE) and lysed by adding $100 \mu \mathrm{L}$ of cell lysis buffer. After inverting the tube to mix, lysis buffer was neutralized by adding $350 \mu \mathrm{L}$ of neutralization solution and mixed by inversion several times. All of the following centrifugation steps in this protocol occurred at maximum speed $(16,000 \mathrm{rcf})$. Samples were centrifuged for 3 min to pellet debris and the supernatant was transferred to the PureYield Minicolumn. Columns were spun for 30 seconds and the flow through was discarded. The columns were washed with $200 \mu \mathrm{L}$ of endotoxin removal wash and spun for $30 \mathrm{~s}$. Wash was discarded and the column was 
washed again with $400 \mu \mathrm{L}$ of column wash solution, column was spun for $30 \mathrm{~s}$. Again, the wash was discarded and the column was transferred to a clean $1.5 \mathrm{~mL}$ Eppendorf tube for elution. Sixty microliters of sterile TE buffer was added to the membrane of the column and incubated at room temperature for $5 \mathrm{~min}$. The column was then spun for $30 \mathrm{~s}$ to elute DNA.

Following preparation of the plasmids they were sent for sequencing analysis by Sequetech (Mountain View, CA) to confirm that mutagenesis was correct.

\section{Maxi Prep Plasmid Purification}

Bacterial cultures were grown overnight at $37^{\circ} \mathrm{C}$, shaking at $225 \mathrm{rpm}$. Cultures were centrifuged the following morning at $5000 \mathrm{rpm}$ for $10 \mathrm{~min}$. The pellets were resuspended in $10 \mathrm{~mL}$ of cold resuspension buffer (Qiagen Buffer P1) with RNase added. Ten $\mathrm{mL}$ of lysis buffer (Qiagen Buffer P2) was added and the tube was inverted 6-7 times to ensure complete lysis of all bacterial cells. Lysis buffer was neutralized by adding 10 $\mathrm{mL}$ of nuetralization buffer (Qiagen Buffer P3) and the tube was inverted until the mixture was homogenous. Cell lysates were incubated on ice for $20 \mathrm{~min}$ prior to centrifugation at $15,000 \mathrm{rpm}$ for $10 \mathrm{~min}$ at $4^{\circ} \mathrm{C}$. During centrifugation, Epoch columns were equilibrated with $30 \mathrm{~mL}$ of Qiagen Buffer QBT which was allowed to pass through the column by gravity flow. Following centrifugation, the supernatant was poured into column and allowed to pass through by gravity flow. Columns were then washed twice with $30 \mathrm{~mL}$ of Buffer QC and purified plasmid DNA was eluted with $15 \mathrm{~mL}$ of Qiagen Buffer QF. 
Plasmid DNA was precipitated by adding $12.5 \mathrm{~mL}$ of $100 \%$ isopropanol. The tube was inverted several times to mix and centrifuged at $4{ }^{\circ} \mathrm{C}$ and $15,000 \mathrm{rpm}$ for $30 \mathrm{~min}$. The supernatant was discarded and pelleted DNA was washed in $10 \mathrm{~mL}$ of ice cold $70 \%$ ethanol. The DNA-ethanol mixture was then centrifuged at $4{ }^{\circ} \mathrm{C}$ and $15,000 \mathrm{rpm}$ for 30 min. Ethanol was discarded and the DNA pellet was allowed to air dry for 15 min. Purified plasmid DNA was then resuspended in $1 \mathrm{~mL}$ of sterile Tris-EDTA (TE) Buffer.

\section{Transfection of Mammalian Cells}

Cells were plated $24 \mathrm{~h}$ prior to transfection at the cell density outlined in Table 2 and maintained in complete DMEM. Twenty-four hours after plating, DNA-lipid transfection mixtures were prepared and cells were transfected using Lipofectamine 2000. Plasmid DNA and Opti-MEM were mixed independently of the Lipofectamine 2000: Opti-MEM master mix. Both solutions were incubated at room temperature for 5 min. Next Lipofectamine 2000 master mix was aliquoted to the DNA-Opti-MEM mixture at a 1:1 ration and incubated at room temperature for $20 \mathrm{~min}$. During this incubation period, complete DMEM on cells is replaced with Opti-MEM. The Lipofectamine transfection mixture was then added to each well. Cells were collected 48 h post-transfection.

Table 2: Lipofectamine 2000 transfection cell density

\begin{tabular}{|c|c|c|c|c|c|}
\hline \multirow[b]{2}{*}{ Plate } & \multicolumn{3}{|c|}{ Number of cells } & \multirow{2}{*}{$\begin{array}{c}\text { Lipofectamine } \\
2000(\mu L): \\
\text { Opti-MEM }(\mu L)\end{array}$} & \multirow{2}{*}{$\begin{array}{c}\text { Total [DNA] } \\
(\mathbf{n g} / \mu \mathrm{L}): \\
\text { Opti-MEM }(\mu \mathrm{L})\end{array}$} \\
\hline & HeLa & HEK-293 & HCT116 & & \\
\hline 6 well & $4 \times 10^{5}$ & $5 \times 10^{5}$ & $1 \times 10^{6}$ & $10: 250$ & $4000: 250$ \\
\hline 12 well & $2 \times 10^{5}$ & $2.5 \times 10^{5}$ & $5 \times 10^{5}$ & $4: 100$ & $1600: 100$ \\
\hline 96 well & -- & $2.5 \times 10^{4}$ & -- & $0.5: 25$ & $200: 25$ \\
\hline
\end{tabular}




\section{Degradation Assays}

Degradation of overexpressed MEF2C was tested by transfection MEF2C-HA in the absence and presence of MAML1. In the case of proteasome inhibition, cells were treated with $10 \mu \mathrm{M}$ MG132 or DMSO as a carrier control 24 h post transfection. Cells were collected $48 \mathrm{~h}$ post transfection and analyzed via western blot. MEF2C protein levels were analyzed by densitometry of the western blot and normalized to the glyceraldehyde 3-phosphate dehydrogenase (GAPDH) loading control for each sample. The density of the MEF2C alone sample was normalized to $100 \%$ and all other samples were represented as a percentage of the control. Degradation assays of MEF2C were performed through co-expression of MAML1, MAML2, MAML3 and MAML1 deletion constructs MAML1 1-301, MAML1 $\Delta 75-300$.

\section{MEF2C Immunoprecipitation (IP)}

HEK293 cells were transfected with Myc-MEF2C, Myc-MAML1, HA-Ubiquitin and treated with $10 \mu \mathrm{M}$ MG132 or DMSO as a carrier control $24 \mathrm{~h}$ post transfection. Twenty-four hours later samples are collected in RIPA buffer (50 mM Tris-Cl pH 7.9, $150 \mathrm{mM} \mathrm{NaCl}, 1 \% \mathrm{NP}-40,0.5 \%$ DOC, and $0.1 \% \mathrm{SDS}$ ) along with $1 \mathrm{X}$ halt protease cocktail. Cell lysates were sonicated 3 times in $30 \mathrm{~s}$ pulses at $40 \%$ amplitude and centrifuged at 15,000 rpm for $5 \mathrm{~min}$ to remove cell debris. Lysates were then transferred to clean tubes and $25 \mu \mathrm{L}$ pre-washed Protein A/G Magnetic Beads in RIPA buffer were added. Samples were pre-cleared with $5 \mu \mathrm{g}$ rabbit $\mathrm{IgG}$ rotating at $4^{\circ} \mathrm{C}$ for $1 \mathrm{~h}$. Following pre-clearing, magnetic beads were removed from the sample using the magnetic tube rack and supernatants were transferred to a clean tube. Five micrograms of Myc antibody was 
added to each sample and allowed to rotate at $4{ }^{\circ} \mathrm{C}$ overnight. The following morning, 25 $\mu \mathrm{L}$ pre-washed Protein A/G Magnetic Beads were added, and samples were rotated at $4^{\circ} \mathrm{C}$ for $1 \mathrm{~h}$ to IP. The samples containing beads were then washed three times for $5 \mathrm{~min}$ each, in RIPA buffer. All RIPA buffer was removed from tubes and $75 \mu \mathrm{L} 2 \mathrm{X}$ Laemmli buffer (4\% SDS, 20\% glycerol, 10\% 2-mercaptoethanol, $0.004 \%$ bromphenol blue and $0.125 \mathrm{M}$ Tris $\mathrm{HCl} \mathrm{pH} 6.8$ ) was added to each tube. Tubes were placed on a $95^{\circ} \mathrm{C}$ heat block for $7 \mathrm{~min}$ to denature the proteins and prepare samples for SDS-PAGE.

\section{SDS-PAGE Preparation and Western Blotting}

To prepare 10\% SDS-PAGE gels the separating gel containing $2.5 \mathrm{~mL} 40 \%$ acrylamide, $2.5 \mathrm{~mL}$ 4X Lower buffer, $5 \mathrm{~mL}$ MilliQ water, $60 \mu \mathrm{L}$ 10\% Ammonium Persulfate (APS) and $15 \mu \mathrm{L}$ TEMED, was poured and allowed to solidify for $30 \mathrm{~min}$. Next the stacking gel was prepared with $525 \mu \mathrm{L} 40 \%$ acrylamide, $1.25 \mathrm{~mL} 4 \mathrm{X}$ Upper buffer, $3.225 \mathrm{~mL}$ MilliQ water, $25 \mu \mathrm{L} \mathrm{10 \%}$ APS and $6 \mu \mathrm{L}$ TEMED. The stacking gel was allowed to solidify for $1 \mathrm{~h}$ prior to usage or storage.

Eight percent SDS-PAGE gels were used for IP experiments and made with the following modifications to the separating gel: $2.0 \mathrm{~mL} 40 \%$ acrylamide, and $5.5 \mathrm{~mL}$ MilliQ water were used. All other reagents remained consistent.

Western gels were run in the Mini-PROTEAN gel apparatus at $100 \mathrm{~V}$ for 15 minutes, the voltage was then increased to $200 \mathrm{~V}$ for $45 \mathrm{~min}$ or until the dye front ran off the bottom of the gel. To transfer SDS-PAGE gels to PVDF membrane, membrane was prepared by soaking in $100 \%$ methanol followed by soaking in transfer buffer. Following set up of the transfer cassette, the transfer was run at $100 \mathrm{~V}$ for $1 \mathrm{~h}$ at $4^{\circ} \mathrm{C}$. Following 
transfer, the membrane was blocked in 5\% Dry Milk in TBS-T for $1 \mathrm{~h}$. The Blocking reagent was washed off in TBS-T and primary antibody was added at a dilution of 1:1000 in 5\% milk overnight. The following day primary antibody was washed off 3 times for 15 min each in TBS-T. Species-specific secondary antibody conjugated to Horse radish peroxidase (HRP) were added to membranes at a dilution of 1:10,000 and rocked for 1.5 h. The secondary antibody was washed 3 times for 10 min each in TBS-T. The protein molecular weight marker was then marked with pencil and the membrane was sprayed with ECL (HyGLO). Western blots were imaged using the Image Quant imaging system.

\section{Promoter Assays}

HEK293 cells were plated in a 96-well dish and transfected with the MEF2CLuc (MEF2C-Luciferase promoter), pRL-TK (renilla), MAML1, and MEF2C expression plasmids according to the protocol described above. Samples were collected $48 \mathrm{~h}$ post transfection in $25 \mu \mathrm{L} 1 \mathrm{X}$ Passive Lysis Buffer and shaken for 15 min. Stop \& Glo reagent (renilla) was prepared from 50X Stop \& Glo substrate and Stop \& Glo buffer. Luciferase reagent was prepared in advance and stored at $-80^{\circ} \mathrm{C}$ to be thawed upon use. After cells were completely lysed, the luminescence was read on the luminometer under the following conditions: $50 \mu \mathrm{L}$ Luciferase reagent, $50 \mu \mathrm{L}$ Stop \& Glo reagent, $5 \mathrm{sec}$ delay between injection and measure, $10 \mathrm{sec}$ integration time. Firefly luciferase and renilla readings were obtained and analyzed using Microsoft Excel. 


\section{RESULTS}

\section{Co-expression of MEF2C and Mastermind-like 1 Leads to Reduced MEF2C Protein Levels}

Co-transfection of MEF2C and MAML1 in HEK293 cells resulted in decreased MEF2C protein levels $48 \mathrm{~h}$ post transfection compared to MEF2C alone. MEF2C expressed alone had a darker band, indicating more protein is present. Lane 3 of Figure 3 A shows a visible decrease in the intensity of MEF2C when co-transfected with MAML1. These results are consistent with preliminary data produced by our lab and this suggests that MAML1 was able to induce degradation of MEF2C in our over-expressed cell culture system.

A

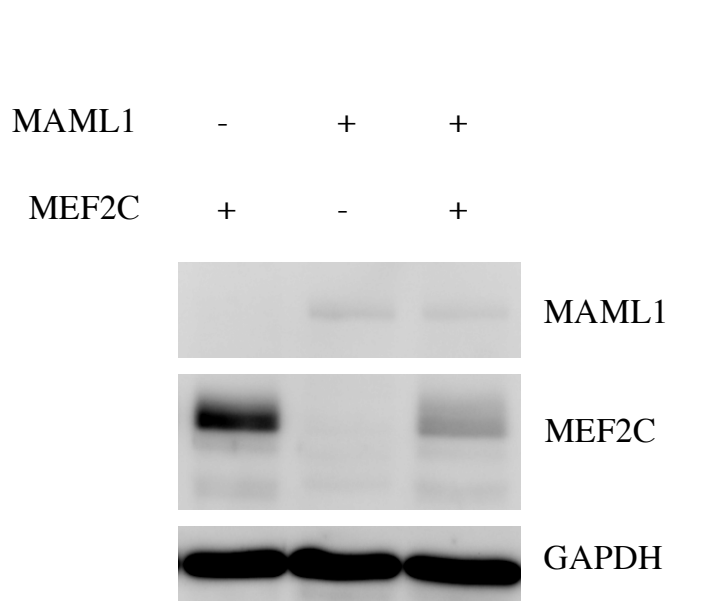

B

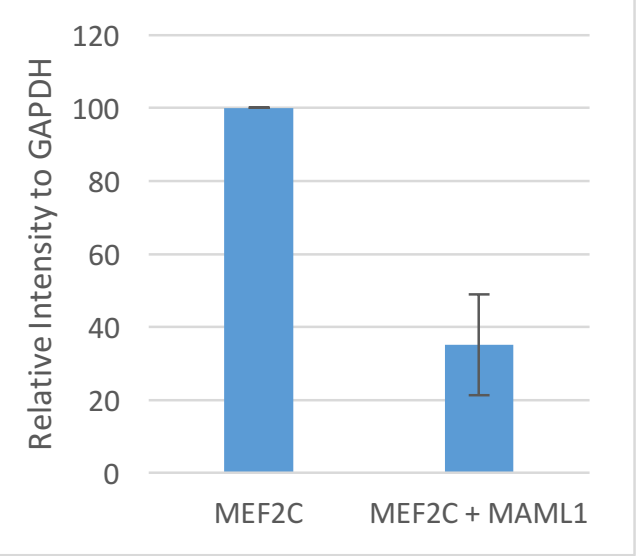

Figure 3. MAML1 induced degradation of MEF2C. HEK293 cells transfected with MEF2C alone, MAML1 alone, and MEF2C and MAML1 together. (A) Western blot of HEK293 protein samples, stained for Myc tagged MAML1, Myc tagged MEF2C, and GAPDH. (B) Densitometry analysis of A. MEF2C protein levels are quantified and normalized to GAPDH. MEF2C alone is normalized 100 and all other samples are shown as a percent of the original. Representative of 5 independent experiments. 


\section{Both TADs of Mastermind-like 1 are Required for Degradation to Occur}

To determine which domains of MAML1 are required to induce degradation of MEF2C, MEF2C expression plasmid were co-transfected with two MAML1 deletion constructs, MAML1 1-301 and MAML1 $\Delta 75-300$. MAML1 1-301 contains the first 301 amino acids, lacking the $\mathrm{C}$ terminus TAD2 and acidic domain 2. MAML1 $\triangle 75-300$ has amino acids 75-300 deleted, lacking TAD1 and the first acidic domain (Figure 4).

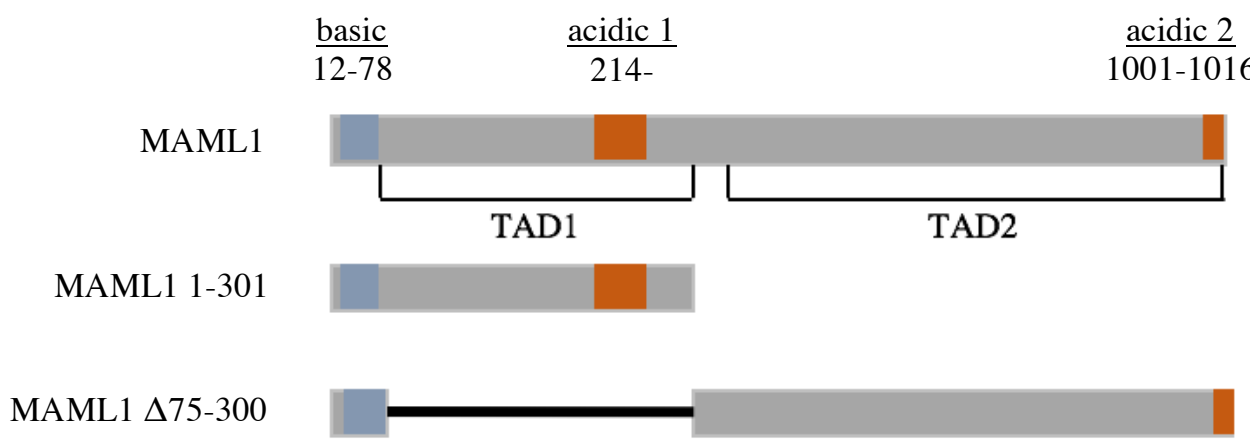

Figure 4. MAML1 deletion construct deficient in TAD1 or TAD2. MAML1 1-301 contains the $\mathrm{N}$ terminal basic domain and acidic domain 1 along with TAD1. MAML1 $\Delta 75-300$ has the $\mathrm{N}$ terminal basic domain and acidic domain 2, but has a TAD1 deletion.

Neither deletion construct induced degradation of $\mathrm{MEF} 2 \mathrm{C}$ to the levels of wild type MAML1. However, MAML1 $\triangle 75-300$ consistently showed a decrease in MEF2C protein levels compared to MAML1 1-301. These results suggest that regions within both TADs are required to induce full degradation of MEF2C. Interestingly, cotransfection with MAML1 1-301 showed an increase in MEF2C protein levels above that of $\mathrm{MEF} 2 \mathrm{C}$ alone. While this phenomenon may have been due to transfection error, it is possible that TAD1 of MAML1 stabilized overexpressed MEF2C. 
A
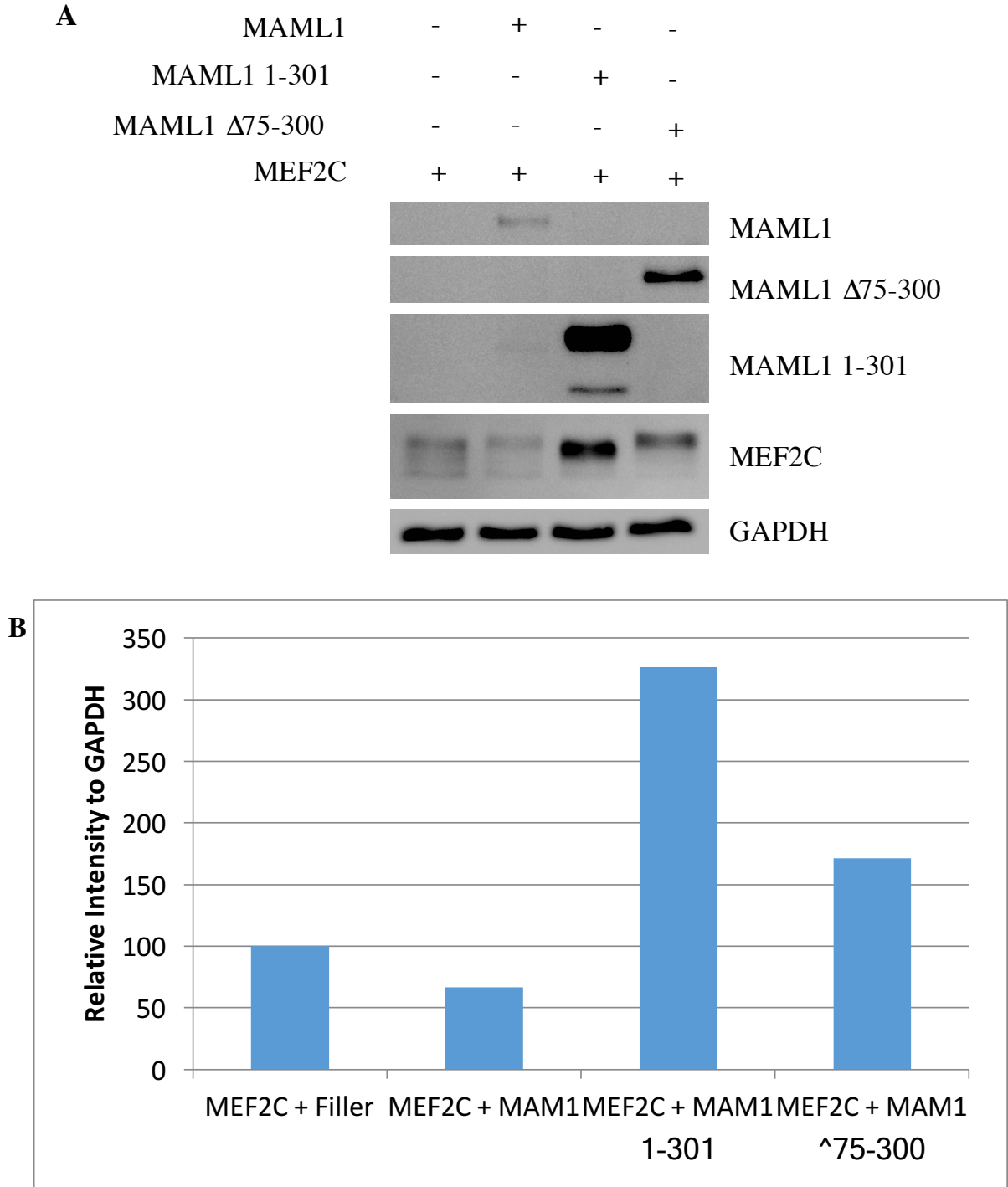

Figure 5. Co-expression of MEF2C with MAML1 deletion constructs. HEK293 cells transfected with MEF2C alone, MEF2C and wild type MAML1, MEF2C with MAML1 1-301, and MEF2C with MAML1 $475-300$. (A) Western blot stained for Myc tagged MAML1 constructs, HA tagged MEF2C, and GAPDH. (B) Densitometry of analysis of A. MEF2C protein levels normalized to GAPDH first. Represents 1 experiment.

\section{Mastermind-like 2 and and Mastermind-like 3 Elicit Varying Effects on MEF2C Protein Levels}

MAML2 and MAML3 are members of the mastermind-like family capable of inducing degradation of NICD (15). Due to this ability, we wanted to examine if MAML2 and 
MAML3 could also induce degradation of MEF2C in vitro. HEK293 cells transfected with MEF2C in the presence and absence of MAML2 did not show reduced MEF2C levels, indicating MAML2 may not be capable of eliciting the same functions as MAML1. Co-expression of MEF2C with MAML3 has shown inconclusive results. In some experiments, MAML3 appeared to induce complete degradation of MEF2C, more so than we have seen with MAML1. However, in recent experiments MAML3 has shown no ability to induce degradation of MEF2C. These contradicting results have not allowed us to conclude whether MAML2 or MAML3 are capable of inducing degradation of MEF2C.

A

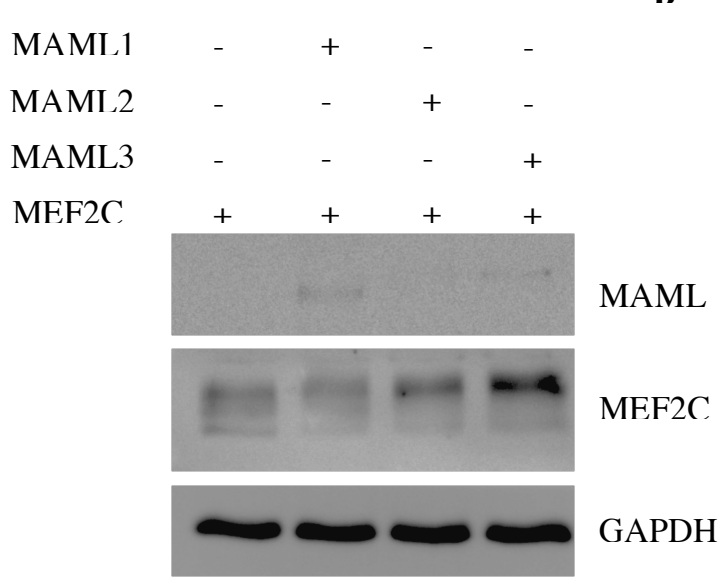

B

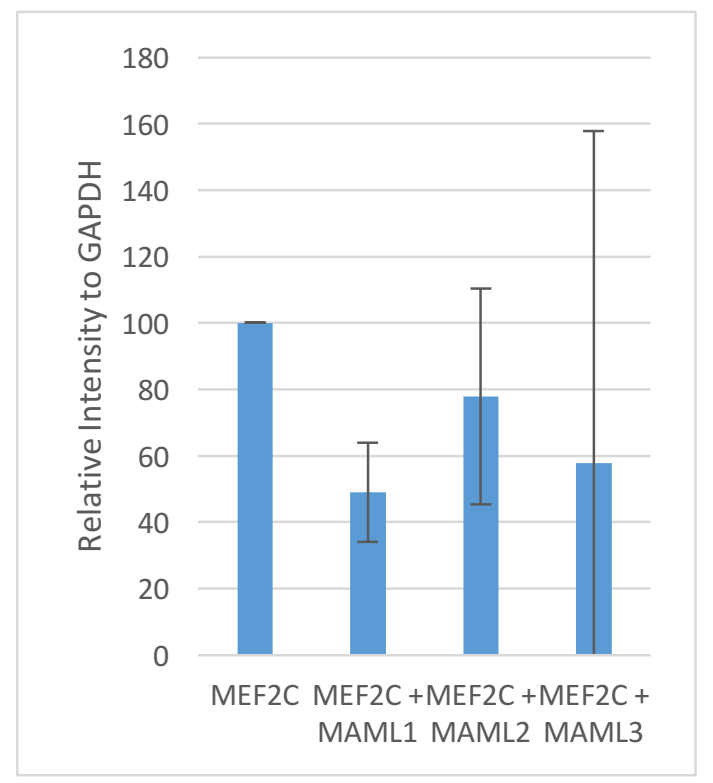

Figure 6. MAML2 and MAML3 do not induce degradation of MEF2C. MEF2C expressed alone or with MAML1, MAML2, or MAML3. (A) MAML1 shows degradation of MEF2C; however, when MEF2C is co-expressed with MAML2 and MAML3 we do not see degradation occurring. GAPDH is a loading control. (B) Quantification of MEF2C protein levels by densitometry analysis. Representative of 3 independent experiments. 


\section{MG132 Treatment Recovers MEF2C Protein Levels}

Inhibition of the 26s proteasome in HEK293 cells transfected with MEF2C and MAML1 with MG132 treatment shows increased protein levels compared to DMSO controls. In order to determine whether MAML1 is facilitating the degradation of $\mathrm{MEF} 2 \mathrm{C}$ through the ubiquitin- proteasome pathway, we treated samples with $10 \mu \mathrm{M}$ MG132. If MEF2C is being ubiquitinated and targeted to the proteasome, by inhibiting this pathway we would expect to see an increase in MEF2C protein levels via Western blot analysis. HEK293 cells expressing MEF2C alone showed an increase in MEF2C protein levels when treated with MG132 (Figure 7). Cells co-expressing MEF2C and MAML1 showed increased MEF2C expression when treated with MG132 compared to cells expressing MEF2C and MAML1 treated with DMSO. However, MG132 did not fully prevent MAML1 induced degradation because MEF2C protein levels are still lower than that of MEF2C alone, both with and without MG132 treatment. MAML1 is regulated through the ubiquitin proteasome pathway and is therefore a good control to indicate whether MG132 is effectively working (43). MAML1 was stabilized by MG132 treatment, indicating MG132 treatment effectively inhibited protein degradation through the $26 \mathrm{~S}$ proteasome (Figure $7 \mathrm{~A}$ ).

HEK293 cells transfected with MEF2C and MAML2 or MAML3 showed increased protein levels after MG132 treatment. Despite seeing minimal degradation when co-expressed with MAML2 and MAML3, MG132 treatment was still capable of stabilizing MEF2C protein levels (Figure 7). 
A

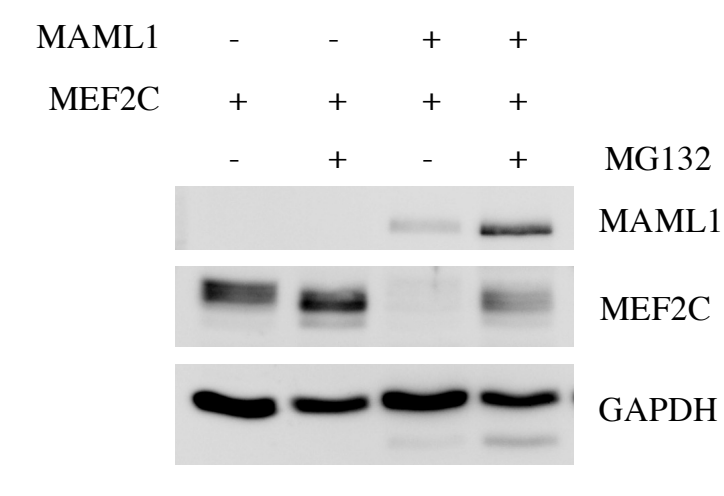

B

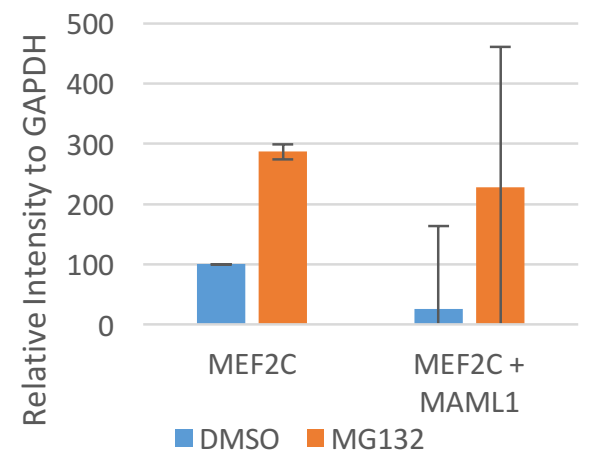

MG132

MAML

MEF2C

GAPDH

Figure 7. MG132 treatment recovers MEF2C protein levels. (A) Samples containing MEF2C alone and MEF2C and MAML1 treated with MG132 show an increase of MEF2C protein levels. (B) Densitometry analysis of MEF2C protein levels in A, normalized to GAPDH show MG132 treatment recovers MEF2C. Representative of 3 independent experiments. (C) MEF2C expressed alone or with MAML1, MAML2, or MAML3. Untreated samples containing MEF2C co-expressed with MAML1, MAML2, and MAML3 show a shift in molecular weight. Treatment with MG132 increases $\mathrm{MEF} 2 \mathrm{C}$ protein levels and shows a slight reduction in molecular weight. 


\section{Detection of Ubiquitin on MEF2C}

To confirm MEF2C is being ubiquitinated and targeted to the 26s proteasome we immunoprecipitated (IP) MEF2C to determine if we could detect HA-tagged ubiquitin as a covalent modification. HEK293 cells transfected with varying combinations of MycMEF2C, Myc-MAML1 and HA-ubiquitin were immunoprecipitated for Myc and western blotted for both Myc and HA. HA-ubiquitin was detected in both MEF2C alone and MEF2C with MAML1 protein extracts treated with MG132. Samples treated with MG132 showed a distinct HA-ubiquitin band at the same molecular weight as MEF2C. However, HA-ubiquitin was not detectable on untreated MEF2C samples.

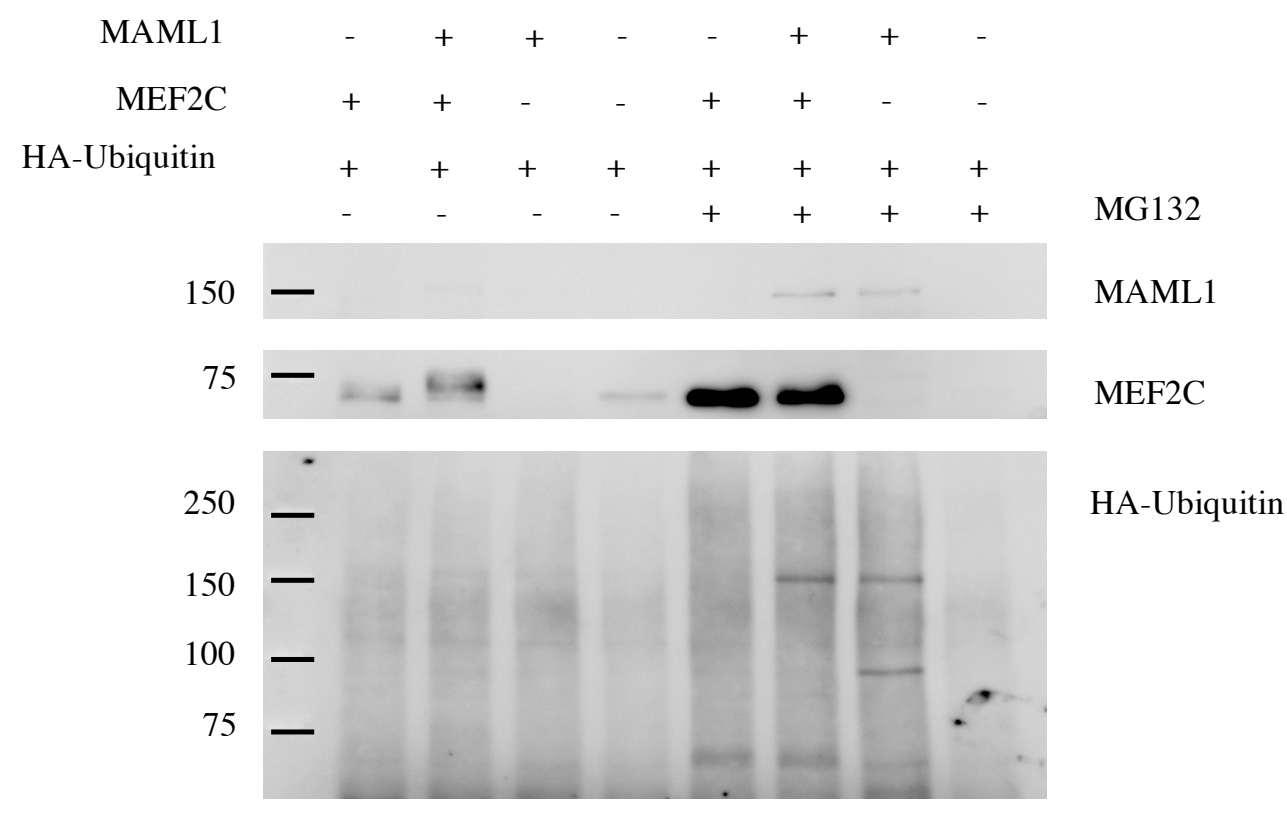

Figure 8. Detection of ubiquitin on MEF2C treated with MG132. Immunoprecipitation of MEF2C with and without MAML1. Samples treated with MG132 show a distinct band at the same molecular weight of MEF2C in the HA-stained western blot. We also detect HA-Ubiquitin on MAML1, which has been previously shown to be ubiquitinated. 
To determine whether MAML1 facilitates the ubiquitination of MEF2C, MycMEF2C IPs with and without MAML1 were compared. 48 Hours post-transfection there was no noticeable difference in the amount of ubiquitin detected on MEF2C with overexpressed MAML1 compared to MEF2C alone. MAML1 may still be responsible for enhancing the ubiquitination of MFE2C, but there may not be a detectable difference at $48 \mathrm{hrs}$. To confirm this, IP's should be performed at varying time points post transfection and the ubiquitin levels should be compared over time.

\section{Mastermind Does Not Induce Degradation of MEF2C in Colorectal Cancer Cells}

To determine whether MAML1 induces the degradation of native MEF2C, the colorectal cancer cell line HCT116 was used. HCT1116 cells stably express MEF2C, allowing us to over express MAML1 and determine the effects on endogenous MEF2C. HCT116 cells transfected with MAML1 showed no detectable decrease in the amount of MEF2C present when normalized to GAPDH (Figure 9). MG132 treatment did increase MEF2C levels, indicating native MEF2C is degraded through the proteasome, despite the inability of MAML1 to induce degradation. 
$\mathbf{A}$

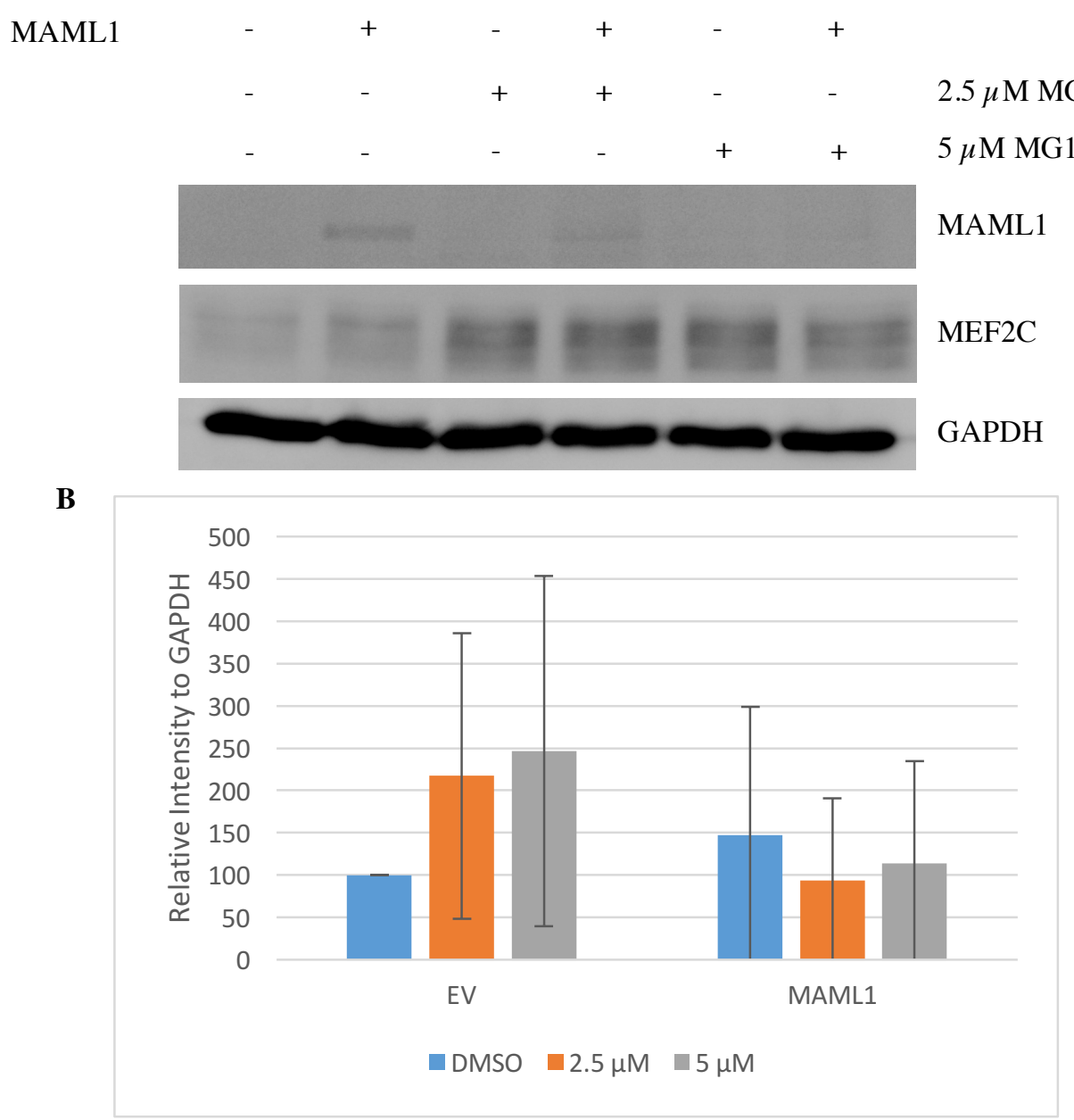

Figure 9. MAML1 does not induce degradation of MEF2C in HCT116 cells. (A) HCT116 cells transfected with MAML1 and western blotted for MEF2C, Myc- MAML1, and GAPDH. (B) Densitometry quantification of A shows MG132 treatment increases MEF2C protein levels, however MAML1 expression does not show degradation of MEF2C. Representative of 3 independent experiments.

Similar to experiments performed with overexpressed MEF2C, the MAML1 deletion constructs (MAML1 1-301 and MAML1 475-300) were tested against native MEF2C in HCT116 cells. Both MAML1 deletion constructs showed no effect on MEF2C stability. 
A

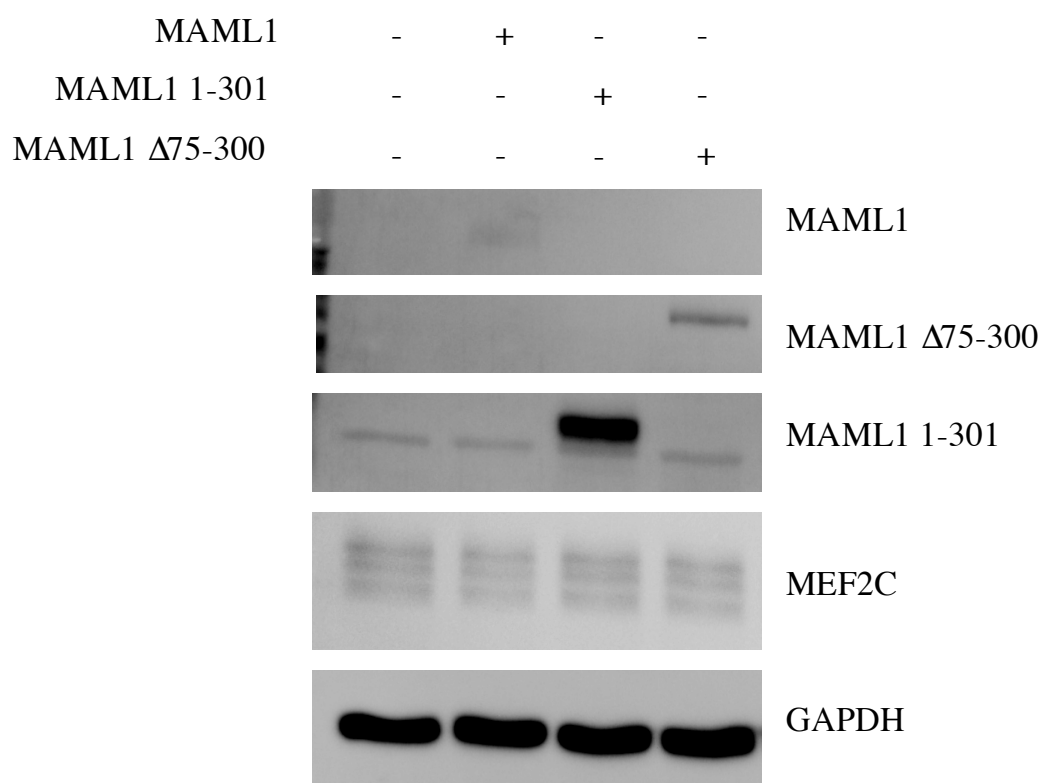

B

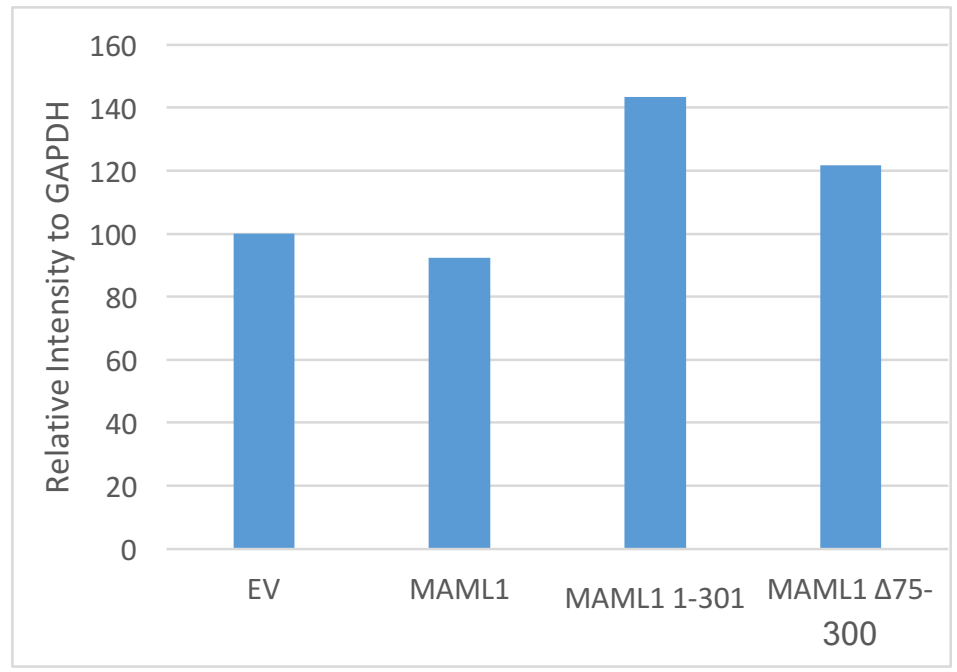

Figure 10. MAML1 deletion constructs are unable to induce degradation of native MEF2C. (A) HCT116 cells transfected with wild type MAML1, MAML1 1-301, or MAML1 $\triangle 75-300$. Show no reduction in MEF2C protein levels. (B) Densitometry analysis of MEF2C protein levels in A normalized to GAPDH. Represents 1 experiment.

MAML2 and MAML3 were tested for their ability to induce degradation of endogenous MEF2C. There was no distinguishable difference in MEF2C protein levels 
in the presence of MAML2 or MAML3. However, samples that were treated with 2.5 $\mu \mathrm{M}$ MG132 did show an increase in MEF2C, consistent with what was previously seen. Indicating endogenous MEF2C is still likely being degraded through the ubiquitinProteasome pathway.

A

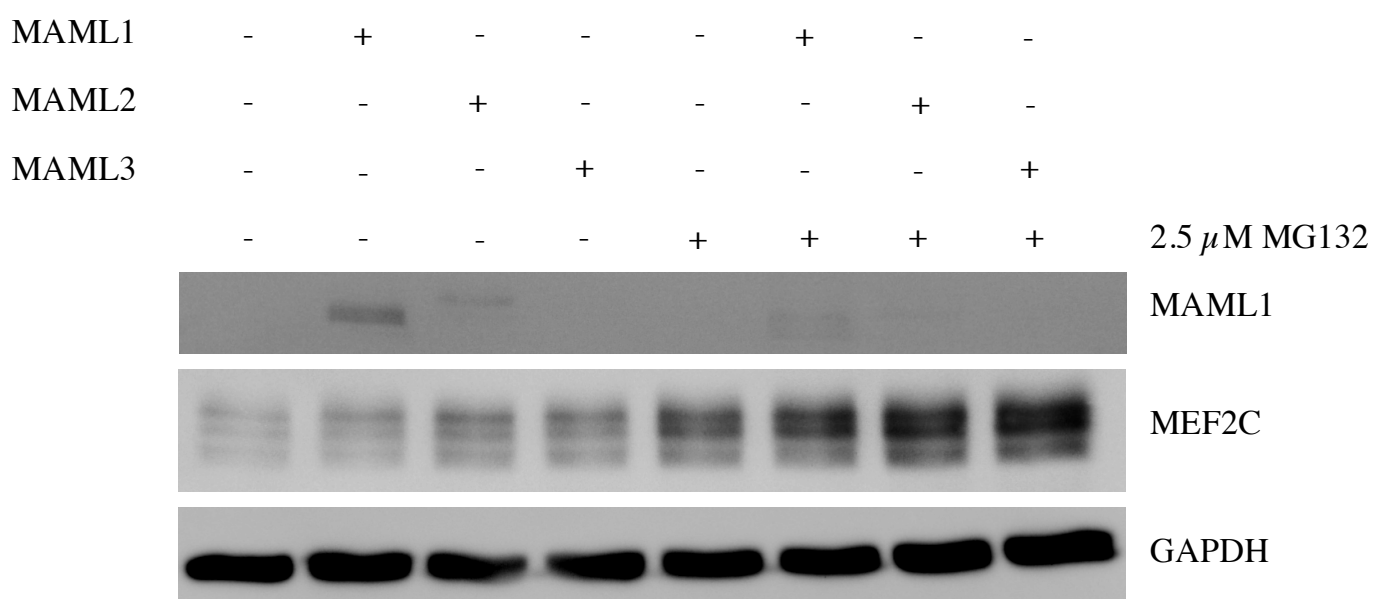

B

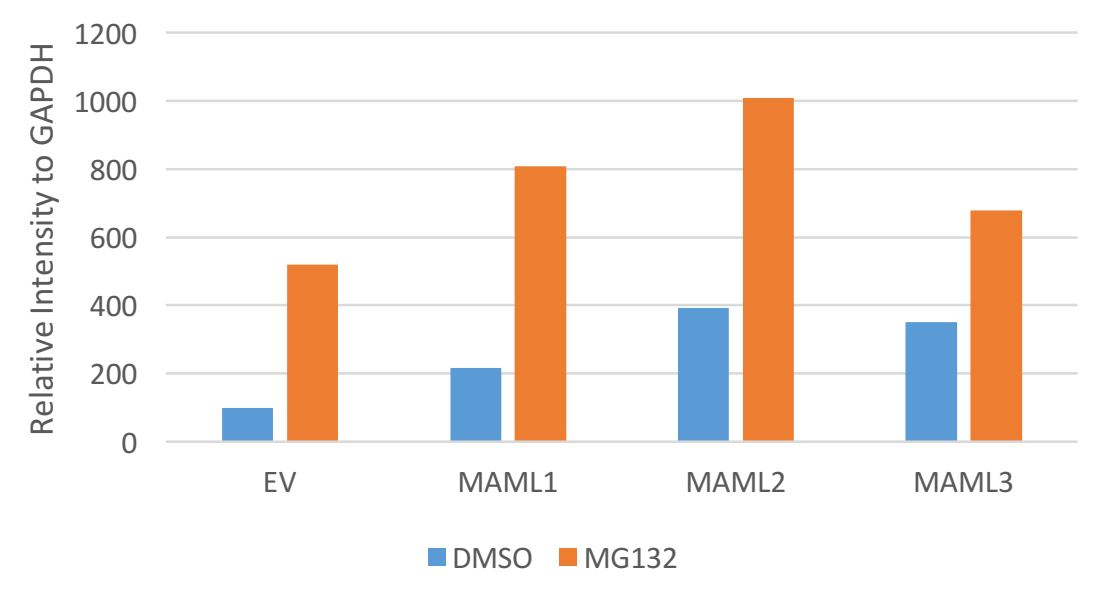

Figure 11. MAML2 and MAML3 are unable to induce degradation of MEF2C in HCT116 cells. (A) HCT116 cells transfected with MAML1, MAML2, and MAML3 show no reduction in MEF2C protein levels, determined by western blot. Cells treated with MG132, regardless of MAML expression, show an increase in MEF2C protein levels. (B) Densitometry analysis of MEF2C levels in HCT116 cells. Represents 1 experiment. 


\section{MEF2C Mutants Do Not Prevent MAML1 Induced Degradation}

Serine 98 (S98) and serine 110 (S110) in MEF2C were previously suggested as potential phosphorylation sites responsible for the ubiquitination of $\operatorname{MEF} 2 \mathrm{C}(37,38)$. To identify if S98 and S110 play a role in MAML1's ability to induce degradation of MEF2C we mutated them to alanine (MEF2C S98A S110A). HEK293 cells transfected with MEF2C S98A S110A showed increased stability compared to wild type MEF2C. Although when co-expressed with MAML1, MEF2C S98A S110A was still degraded. Indicating MAML1 does not rely on S98 or S110 to induce degradation of MEF2C.

$\mathbf{A}$

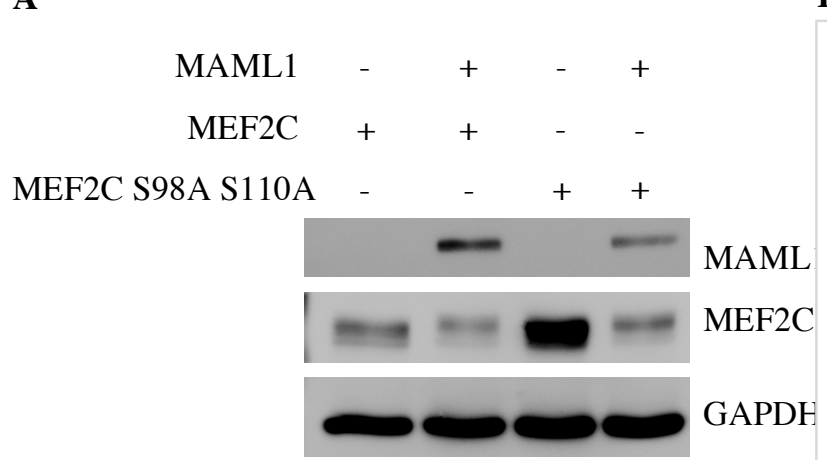

B

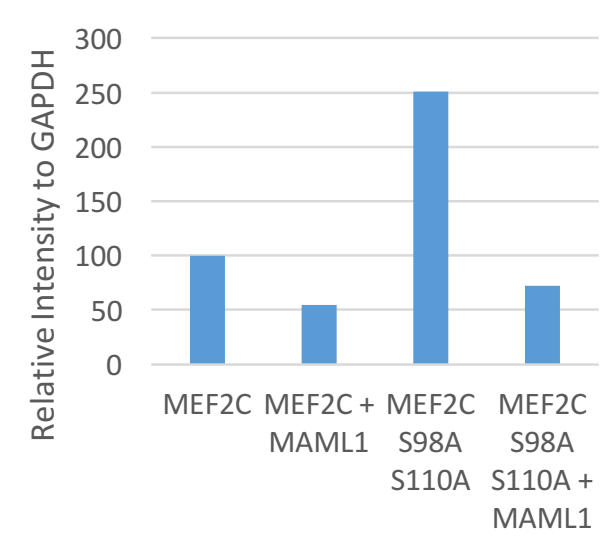

Figure 12. MAML1 can induce degradation of MEF2C S98A S110A. (A) HEK293 cells transfected with wild type MEF2C or MEF2C S98A S110A when co-expressed with MAML1 still show a reduction in MEF2C protein levels. (C) Quantified MEF2C protein levels by densitometry analysis normalized to GAPDH. Representative of a single experiment.

\section{Effects on MEF2C Target Promoter Activity}

MAML1 has been previously shown to be a co-activator for MEF2C and is capable of activating the artificial MEF2 luciferase promoter (MEF2Luc) (8). To confirm the functionality MAML1 induced MEF2C degradation we overexpressed 
MEF2Luc and renilla with varying combinations of MEF2C and MAML1. Cells were treated with MG132 or DMSO 24 hours post transfection to determine if inhibiting the degradation pathway increases activity of the target promoter. We found that samples treated with MG132 showed a significantly larger fold change in activation of the target promoter (Figure 13). Additionally, we see an increase in activation in samples containing MAML1 treated with MG132. Because MAML1 is degraded through the ubiquitin- proteasome pathway, MG132 treatment increases the level of both MEF2C and MAML1 in the sample and therefore drastically increasing activation of the MEF2Luc promoter. 
A

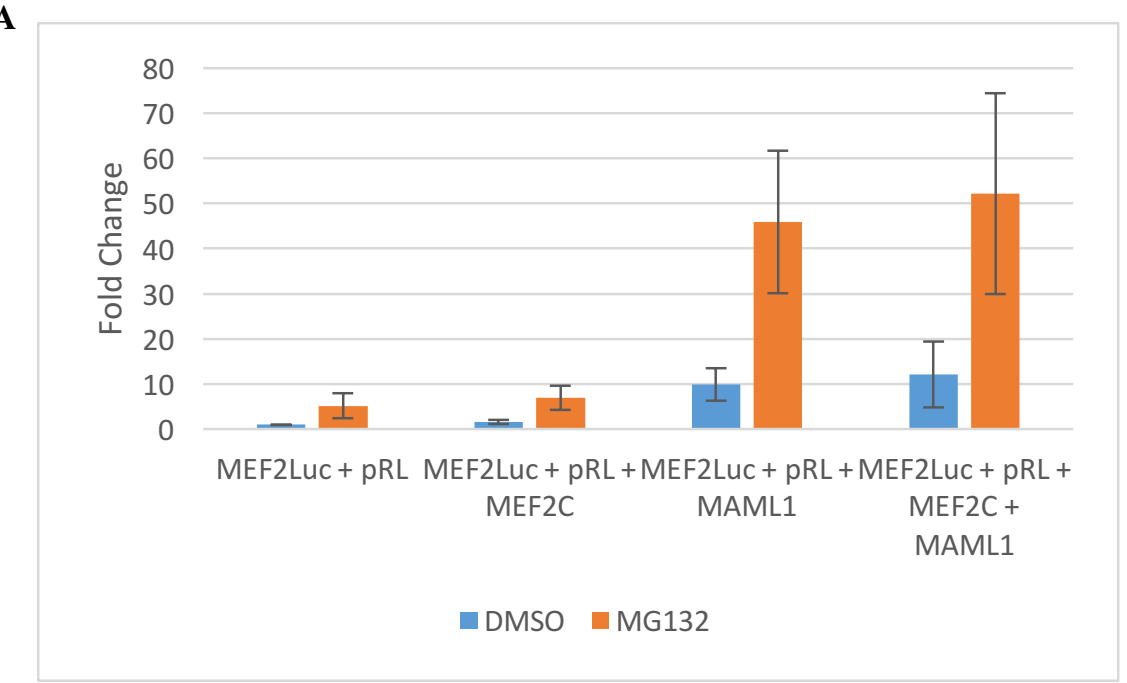

B

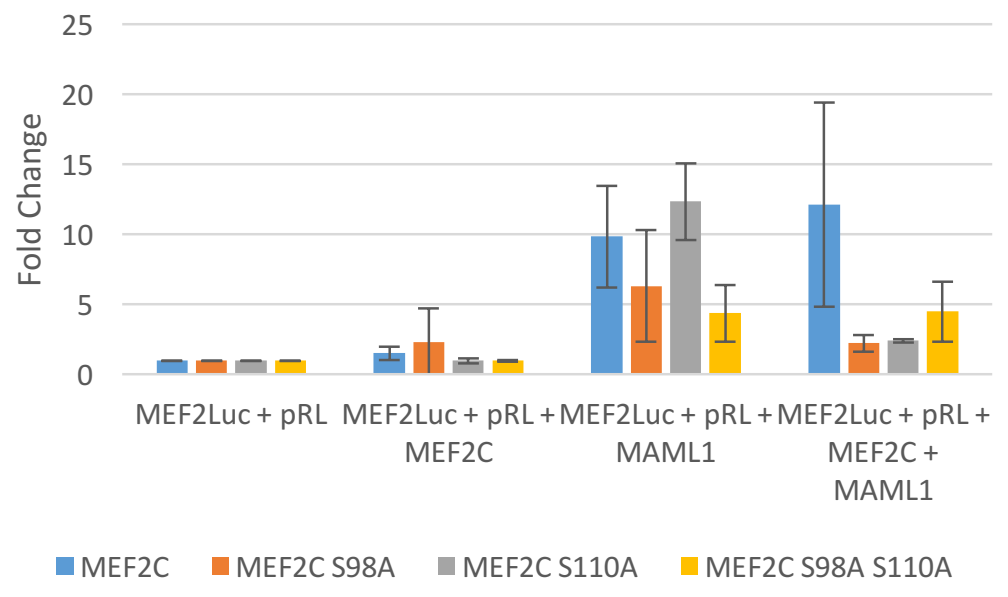

Figure 13. Effects on MEF2 target promoter activity. (A) Luciferase assay of MEF2Luc promoter activity. Displayed as fold change compared to MEF2Luc + pRL samples only. Samples treated with MG132 show a large increase in promoter activity, suggesting MEF2C and MAML1 were not degraded. Represented as averages of 3 experiments. (B) Luciferase assay monitoring MEF2Luc promoter activity using wild type MEF2C, MEF2C S98A, MEF2C S110A, and MEF2C S98A S110A. Represented as averages from 3 independent experiments. 


\section{DISCUSSION}

Understanding the mechanisms that regulate transcription factors is an important aspect to understanding gene expression, development pathways, and disease progression. Many diseases, including numerous types of cancer, develop as a result of abnormalities in gene expression $(12,29,44)$. The ubiquitin-proteasome pathway is exceptionally important in maintaining basal levels of gene expression through the degradation of unneeded transcription factors. This process allows for increased sensitivity in response to external and internal signals, by limiting the response time of target transcription. MAML1 plays a critical role in the co-activation and regulation across multiple signaling pathways. Developing a better understanding of MAML1's function in MEF2C signaling will allow us to better understand its relationship to other processes.

Our results indicate MAML1 facilitates the degradation of overexpressed MEF2C. Here we that show co-expression of MEF2C with MAML1 results in increased degradation of MEF2C. Taken together with the cyclohexamide pulse chase data previously generated by our lab, we can conclude MAML1 is responsible for MEF2C's increased rate of degradation. The specific domain required to induce degradation of MEF2C has not yet been identified. We show that both TAD1 and TAD2 are required for full degradation to occur. However, co-expression of MEF2C with MAML1 lacking TAD1 (MAML1 D75-300) showed decreased protein levels compared to MAML1 1-301 (Figure 5). This suggests that TAD1 and TAD2 do not evoke the same response on MEF2C and TAD1 may be important in stabilizing protein levels. Fryer et al. previously 
showed MAML1 lacking the C-terminus is unable to bind CDK8 and induce degradation of NICD, confirming that TAD2 is required for degradation to occur (10). Because we see the same trend in MEF2C, it is possible that MAML1's association with CDK8 may be responsible for phosphorylation-dependent degradation of MEF2C. However, we were unable to determine if MAML1 increases the amount of ubiquitin detected on MEF2C.

MEF2C is ubiquitinated and degraded by the $26 \mathrm{~S}$ proteasome. It is important to note that ubiquitin is not detectable on samples not treated with MG132. This may be because overexpression of ubiquitin concurrent with overexpression of MEF2C can increase the amount of ubiquitination occurring and therefore increase the rate of degradation in untreated samples. Inhibiting the proteasome allows us to detect HAubiquitin on MEF2C. While our results confirm that MEF2C is ubiquitinated and being degraded by the proteasome, we saw no detectable difference in the level of ubiquitin detected on MEF2C in the presence of MAML1. However, we did see recovery of MEF2C protein levels in the presence of MAML1 when treated with the proteasome inhibitor, MG132. Taken together, these results indicate that MAML1 is likely facilitating the ubiquitin-dependent degradation of MEF2C. However, we are unable to detect a noticeable difference in ubiquitin levels 48 hours post transfection.

Despite confirming that MEF2C is ubiquitinated, we were unable to support previous data suggesting serine 98 and 110 are the primary targets of phosphorylationdependent ubiquitination $(37,38)$. While S98 and S110 may be essential for the turnover of MEF2C in response to cell cycle cues, our results show that it does not prevent MAML1-induced degradation (37). This suggests that MAML1 may be inducing 
degradation of $\mathrm{MEF} 2 \mathrm{C}$ in response to alternate cellular signals. Interestingly, no promoter studies have been published with MEF2C S98 and S110 mutants. Our results show that the MEF2C double mutant is unable to activate transcription of the target promoter compared to wild type controls. If degradation was prevented by S98A and S110A mutations, we would expect to see an increase in promoter activity over wild type MEF2C. Alternatively, S98 and S110 may be involved in activation of the target promoter and unable to initiate transcription. Though we are uncertain of the cause of this phenomenon, S98A and S110A mutations completely reduce activation of the MEF2 target promoter. Further studies need to be performed to identify the function of S98 and S110 in MEF2C-targeted transcription.

While most of our studies used MEF2C expression plasmids in HEK293 cells, we wanted to confirm our results in cells expressing native MEF2C. The colorectal cancer cell line, HCT116, stably expresses MEF2C. Unlike HEK293 cells transfected with MEF2C, HCT116 cells overexpressing MAML1 did not show decreased native MEF2C protein levels. We can speculate that HCT116 cells already express low levels of MAML1, therefore over expression of the protein does not have an effect on MEF2C stability. One way to confirm that MAML1 has no effect on native MEF2C would be to perform an siRNA knock down of MAML1 in HCT116 cells and determine if reconstituted MAML1 is capable of inducing degradation. Despite MEF2C being consistently expressed in HCT116 cells, it does not indicate that the signaling pathway responsible for assembly of the MEF2C transcriptional complex is always active. Therefore, MAML1-induced degradation of MEF2C may rely on an additional stimulus 
to allow for transcription of MEF2C target genes and ultimately increased regulation of the transcription factor.

It is possible that MEF2C may be a more ubiquitous transcription factor, functioning across multiple regulatory pathways than it was originally implicated in. The protein interactions associated with MEF2C during a specific cellular event may be responsible for determining the mechanism of degradation. Therefore, MEF2C may have multiple pathways regulating its turnover. More studies need to be done to determine the functionality and specificity of MEF2C regulation across various cellular events and signals. Ultimately, our results indicate that MAML1 induces degradation of MEF2C in a proteasome-dependent degradation pathway independent of S98 and S110 in MEF2C. However, we have yet to identify additional co-factors in the transcriptional complex required for this association to occur. 


\section{REFERENCES}

1. Leifer, D., Krainc, D., Yu, Y. T., McDermott, J., Breitbart, R. E., Heng, J., Neve, R. L., Kosofsky, B., Nadal-Ginard, B., and Lipton, S. a (1993) MEF2C, a MADS/MEF2family transcription factor expressed in a laminar distribution in cerebral cortex. Proc. Natl. Acad. Sci. U. S. A. 90, 1546-1550

2. Leifer, D., Golden, J., and Kowall, N. W. (1994) Myocyte-specific enhancer binding factor 2C expression in human brain development. Neuroscience. 63, 1067-1079

3. Zhang, M., Zhu, B., and Davie, J. (2015) Alternative splicing of MEF2C pre-mRNA controls its activity in normal myogenesis and promotes tumorigenicity in rhabdomyosarcoma cells. J. Biol. Chem. 290, 310-324

4. Xu, Z., Yoshida, T., Wu, L., Maiti, D., Cebotaru, L., and Duh, E. J. (2015)

Transcription Factor MEF2C Suppresses Endothelial Cell Inflammation via Regulation of NF-??B and KLF2. J. Cell. Physiol. 230, 1310-1320

5. Hu, J., Verzi, M. P., Robinson, A. S., Tang, P. L.-F., Hua, L. L., Xu, S.-M., Kwok, P.Y., and Black, B. L. (2015) Endothelin signaling activates Mef2c expression in the neural crest through a MEF2C-dependent positive-feedback transcriptional pathway. Development. 10.1242/dev.126391

6. Pagiatakis, C., Gordon, J. W., Ehyai, S., and McDermott, J. C. (2012) A novel RhoA/ROCK-CPI-17-MEF2C signaling pathway regulates vascular smooth muscle cell gene expression. J. Biol. Chem. 287, 8361-8370

7. Debnath, I., Roundy, K. M., Pioli, P. D., Weis, J. J., and Weis, J. H. (2013) Bone marrow-induced Mef2c deficiency delays B-cell development and alters the expression of key B-cell regulatory proteins. Int. Immunol. 25, 99-115

8. Shen, H., McElhinny, A. S., Cao, Y., Gao, P., Liu, J., Bronson, R., Griffin, J. D., and $\mathrm{Wu}, \mathrm{L}$. (2006) The Notch coactivator, MAML1, functions as a novel coactivator for MEF2C-mediated transcription and is required for normal myogenesis. Genes Dev. 20, 675-688

9. Wu, L., Aster, J. C., Blacklow, S. C., Lake, R., Artavanis-Tsakonas, S., and Griffin, J. D. (2000) MAML1, a human homologue of Drosophila mastermind, is a transcriptional co-activator for NOTCH receptors. Nat. Genet. 26, 484-489

10. Fryer, C. J., White, J. B., and Jones, K. A. (2004) Mastermind recruits CycC:CDK8 to phosphorylate the Notch ICD and coordinate activation with turnover. Mol. Cell. 16, 509-520

11. Borggrefe, T., and Oswald, F. (2009) The Notch signaling pathway: Transcriptional regulation at Notch target genes. Cell. Mol. Life Sci. 66, 1631-1646 
12. Ranganathan, P., Weaver, K. L., and Capobianco, A. J. (2011) Notch signalling in solid tumours: a little bit of everything but not all the time. Nat Rev Cancer. 11, 338351

13. Zein, I., and White, B. (2014) MAML1 mediates MEF2C degradation independent of ubiquitination (930.5). FASEB J. 28, 930.5-

14. Wu, L., Sun, T., Kobayashi, K., Gao, P., and Griffin, J. D. (2002) Identification of a family of mastermind-like transcriptional coactivators for mammalian notch receptors. Mol. Cell. Biol. 22, 7688-7700

15. Lin, S. E., Oyama, T., Nagase, T., Harigaya, K., and Kitagawa, M. (2002) Identification of new human mastermind proteins defines a family that consists of positive regulators for notch signaling. J. Biol. Chem. 277, 50612-50620

16. Fryer, C. J., Lamar, E., Turbachova, I., Kintner, C., and Jones, K. A. (2002) Mastermind mediates chromatin-specific transcription and turnover of the notch enhancer complex. Genes Dev. 16, 1397-1411

17. McElhinny, a S., Li, J.-L., and Wu, L. (2008) Mastermind-like transcriptional coactivators: emerging roles in regulating cross talk among multiple signaling pathways. Oncogene. 27, 5138-5147

18. Kopan, R. (2014) Notch Signaling. 10.1007/978-1-4939-1139-4

19. del Bianco, C., Vedenko, A., Choi, S. H., Berger, M. F., Shokri, L., Bulyk, M. L., and Blacklow, S. C. (2010) Notch and MAML-1 Complexation do not detectably alter the DNA binding specificity of the transcription factor CSL. PLoS One.

10.1371/journal.pone.0015034

20. Gerhardt, D. M., Pajcini, K. V, Teresa, D., Tu, L., Jain, R., Xu, L., Chen, M. J., Rentschler, S., Shestova, O., Wertheim, G. B., Tobias, J. W., Kluk, M., Wood, A. W., Aster, J. C., Gimotty, P. a, Epstein, J. a, Speck, N., Bigas, A., and Pear, W. S. (2014) The Notch 1 transcriptional activation domain is required for development and reveals a novel role for Notch1 signaling in fetal hematopoietic stem cells. Genes Dev. 28, $576-593$

21. Ribeiro, J., and Wallberg, A. E. (2009) Transcriptional mechanisms by the coregulator MAML1. Curr. Protein Pept. Sci. 10, 570-576

22. Woeltje, K., Jabs, M., and Fischer, A. (2015) Serum Induces Transcription of Hey 1 and Hey 2 Genes by Alk1 but Not Notch Signaling in Endothelial Cells. PLoS One. 10, UNSP e0120547-UNSP e0120547

23. Fischer, A., and Gessler, M. (2007) Delta-Notch-and then? Protein interactions and proposed modes of repression by Hes and Hey bHLH factors. Nucleic Acids Res. 35, $4583-4596$ 
24. Popko-Scibor, A. E., Lindberg, M. J., Hansson, M. L., Holmlund, T., and Wallberg, A. E. (2011) Ubiquitination of Notch1 is regulated by MAML1-mediated p300 acetylation of Notch1. Biochem. Biophys. Res. Commun. 416, 300-306

25. Just Ribeiro, M. Saint, Hansson, M. L., Lindberg, M. J., Popko-Ścibor, A. E., and Wallberg, A. E. (2009) GSK3 $\beta$ is a negative regulator of the transcriptional coactivator MAML1. Nucleic Acids Res. 37, 6691-6700

26. Zhao, Y., Katzman, R. B., Delmolino, L. M., Bhat, I., Zhang, Y., Gurumurthy, C. B., Germaniuk-Kurowska, A., Reddi, H. V., Solomon, A., Zeng, M. S., Kung, A., Hui, M., Gao, Q., Dimri, G., Stanculescu, A., Miele, L., Wu, L., Griffin, J. D., Wazer, D. E., Band, H., and Band, V. (2007) The Notch regulator MAML1 interacts with p53 and functions as a coactivator. J. Biol. Chem. 282, 11969-11981

27. Jin, B., Shen, H., Lin, S., Li, J. L., Chen, Z., Griffin, J. D., and Wu, L. (2010) The mastermind-like 1 (MAML1) co-activator regulates constitutive NF-??B signaling and cell survival. J. Biol. Chem. 285, 14356-14365

28. Wu, L., Maillard, I., Nakamura, M., Pear, W. S., and Griffin, J. D. (2007) The transcriptional coactivator Maml1 is required for Notch2-mediated marginal zone Bcell development. Blood. 110, 3618-3623

29. Kuncharin, Y., Sangphech, N., Kueanjinda, P., Bhattarakosol, P., and Palaga, T. (2011) MAML1 regulates cell viability via the NF-??B pathway in cervical cancer cell lines. Exp. Cell Res. 317, 1830-1840

30. Alves-Guerra, M. C., Ronchini, C., and Capobianco, A. J. (2007) Mastermind-like 1 is a specific coactivator of ??-catenin transcription activation and is essential for colon carcinoma cell survival. Cancer Res. 67, 8690-8698

31. Tian, H., Biehs, B., Chiu, C., Siebel, C. W., Wu, Y., Costa, M., de Sauvage, F. J., and Klein, O. D. (2015) Opposing Activities of Notch and Wnt Signaling Regulate Intestinal Stem Cells and Gut Homeostasis. Cell Rep. 11, 33-42

32. Martin, J. F., Schwarz, J. J., and Olson, E. N. (1993) Myocyte enhancer factor (MEF) 2C: a tissue-restricted member of the MEF-2 family of transcription factors. Proc. Natl. Acad. Sci. U. S. A. 90, 5282-6

33. Li, H., and Capetanaki, Y. (1994) An E box in the desmin promoter cooperates with the E box and MEF-2 sites of a distal enhancer to direct muscle-specific transcription. Embo J. 13, 3580-3589

34. Ornatsky, O. I., and McDermott, J. C. (1996) MEF2 protein expression, DNA binding specificity and complex composition, and transcriptional activity in muscle and nonmuscle cells. J. Biol. Chem. 271, 24927-24933

35. Quattrocelli, M., Costamagna, D., Giacomazzi, G., Camps, J., and Sampaolesi, M. 
(2014) Notch signaling regulates myogenic regenerative capacity of murine and human mesoangioblasts. Cell Death Dis. 5, e1448

36. Conboy, I. M., Conboy, M. J., Smythe, G. M., and Rando, T. A. (2003) Notchmediated restoration of regenerative potential to aged muscle. Science (80-. ). 302, $1575-1577$

37. Badodi, S., Baruffaldi, F., Ganassi, M., Battini, R., and Molinari, S. (2015) Phosphorylation-dependent degradation of MEF2C contributes to regulate G2/M transition. Cell Cycle. 14, 1517-1528

38. Di Giorgio, E., Gagliostro, E., Clocchiatti, A., and Brancolini, C. (2015) The control operated by the cell-cycle machinery on MEF2s stability contributes to the downregulation of CDKN1A and the entry in S phase. Mol. Cell. Biol. 35, MCB.01461-14

39. Nawaz, Z., and O'Malley, B. W. (2004) Urban renewal in the nucleus: is protein turnover by proteasomes absolutely required for nuclear receptor-regulated transcription? Mol. Endocrinol. 18, 493-499

40. Deshaies, R. J., and Joazeiro, C. a P. (2009) RING domain E3 ubiquitin ligases. Annu. Rev. Biochem. 78, 399-434

41. Dennis, A. P., and O'Malley, B. W. (2005) Rush hour at the promoter: How the ubiquitin-proteasome pathway polices the traffic flow of nuclear receptor-dependent transcription. J. Steroid Biochem. Mol. Biol. 93, 139-151

42. Geng, F., Wenzel, S., and Tansey, W. P. (2012) Ubiquitin and Proteasomes in Transcription. Annu. Rev. Biochem. 81, 177-201

43. Farshbaf, M., Lindberg, M. J., Truong, A., Bevens, Z., Chambers, E., Pournara, A., Wallberg, A. E., and White, J. B. (2015) Mastermind-like 1 is ubiquitinated: Functional consequences for notch signaling. PLoS One. 10, 1-19

44. Vogelstein, B., Papadopoulos, N., Velculescu, V. E., Zhou, S., Diaz Jr., L. A., and Kinzler, K. W. (2013) Cancer Genome Landscapes. Science (80-. ). 339, 1546-1558 